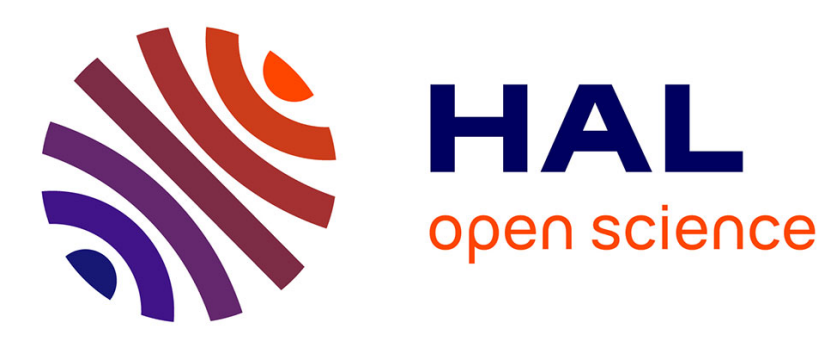

\title{
Numerical analysis of the nonlinear Schrödinger equation with white noise dispersion
}

\author{
Radoin Belaouar, Anne de Bouard, Arnaud Debussche
}

\section{To cite this version:}

Radoin Belaouar, Anne de Bouard, Arnaud Debussche. Numerical analysis of the nonlinear Schrödinger equation with white noise dispersion. Stochastics and Partial Differential Equations: Analysis and Computations, 2015, 3 (1), pp.103-132. 10.1007/s40072-015-0044-z * hal-00948570

\author{
HAL Id: hal-00948570 \\ https://hal.science/hal-00948570
}

Submitted on 18 Feb 2014

HAL is a multi-disciplinary open access archive for the deposit and dissemination of scientific research documents, whether they are published or not. The documents may come from teaching and research institutions in France or abroad, or from public or private research centers.
L'archive ouverte pluridisciplinaire HAL, est destinée au dépôt et à la diffusion de documents scientifiques de niveau recherche, publiés ou non, émanant des établissements d'enseignement et de recherche français ou étrangers, des laboratoires publics ou privés. 


\title{
NUMERICAL ANALYSIS OF THE NONLINEAR SCHRÖDINGER EQUATION WITH WHITE NOISE DISPERSION
}

\author{
RADOIN BELAOUAR, ANNE DE BOUARD, AND ARNAUD DEBUSSCHE
}

\begin{abstract}
This article is devoted to the numerical study of a nonlinear Schrödinger equation in which the coefficient in front of the group velocity dispersion is multiplied by a real valued Gaussian white noise. We first perform the numerical analysis of a semi-discrete Crank-Nicolson scheme in the case when the continuous equation possesses a unique global solution. We prove that the strong order of convergence in probability is equal to one in this case. In a second step, we numerically investigate, in space dimension one, the behavior of the solutions of the equation for different power nonlinearities, corresponding to subcritical, critical or supercritical nonlinearities in the deterministic case. Numerical evidence of a change in the critical power due to the presence of the noise is pointed out.
\end{abstract}

\section{INTRODUCTION}

The cubic nonlinear Schrödinger (NLS) equation is a model describing the propagation of light waves in optical fibers. In some circumstances - this is typically the case in dispersion-managed fibers - the group velocity dispersion may vary along the fiber (see $[1,2])$ and this gives rise in the mathematical model to a NLS equation with a timevarying coefficient in front of the dispersion (Laplace operator). It has been proved in $[18,9]$ (see also [15]) that when this coefficient is random, with mean zero, and under some specific scaling, the limit equation (as the correlation of the noise tends to zero) is a NLS equation with white noise dispersion (i.e. the preceding coefficient becomes a real valued time white noise).

We consider in this paper the more general stochastic nonlinear Schrödinger equation

$$
\left\{\begin{array}{l}
i d u+\Delta u \circ d \beta+|u|^{2 \sigma} u d t=0 \\
u(0)=u_{0}
\end{array}\right.
$$

where the unknown $u$ is a complex valued random process on a probability space $(\Omega, \mathcal{F}, \mathbb{P})$ depending on $t \geq 0$ and $x \in \mathbb{R}^{d}$, and the nonlinear term $|u|^{2 \sigma} u$ is a power term, $\sigma$ being a positive real number. The noise term involves a real valued Brownian motion $\beta$ associated to a filtration $\left(\mathcal{F}_{t}\right)_{t \geq 0}$. The product $\circ$ is a Stratonovich product,

Key words and phrases. Stochastic partial differential equations, numerical analysis, white noise dispersion. 
and equation (1.1) is formally equivalent to its Itô form:

$$
\left\{\begin{array}{l}
i d u+\frac{i}{2} \Delta^{2} u d t+\Delta u d \beta+|u|^{2 \sigma} u d t=0 \\
u(0)=u_{0}
\end{array}\right.
$$

Equation (1.1) has been studied in [9] in the case $\sigma<2 / d$ and in [11] in the case $d=1$ and $\sigma=2$, and the existence of a unique global square integrale solution was proved. However, nothing is known about the qualitative behavior of the solutions of (1.1) note that the Hamiltonian structure of the deterministic NLS equation is destroyed by the presence of a time varying dispersion coefficient. While the case of a deterministic time-periodic coefficient has been investigated from the numerical as well as theoretical point of view (see e.g. $[17,21,22]$ ), few investigations have been performed in the stochastic case (see however [15] and [18] for numerical simulations in the cubic case), and no theoretical result on the qualitative behavior is known.

These considerations motivated us for a more systematic numerical study of equation (1.1) for general values of the parameter $\sigma$. We will be in particular interested in the blow up phenomena. In [10] and [3], one of the authors conducted such investigations for a different stochastic equation, in which the noise, which was there a space-time noise, white in time and possibly correlated in space, acted as a linear potential in the equation. It was then observed that in the supercritical case $\sigma>2 / d$, as the space correlation of the noise tends to zero, the blow up mechanism could be stopped. Note that it was proved in [7] that if on the opposite, the noise is spatially sufficiently correlated, then blow up occurs for any initial data with a positive probability, still in the case $\sigma>d / 2$. We will here do the same investigation in the, somewhat simpler, case of equation (1.1), where the noise only depends on time (see Section 3).

In a first step, we study the order of convergence for equation (1.1), of a semi-discrete Crank-Nicolson scheme, which is one of the schemes used in the numerical simulations of Section 3. As is natural for a Stratonovich noise, we use a mid-point discretization of the noise. The scheme then presents the advantage of preserving the $L^{2}$-norm, which is a conserved quantity of the continuous equation. The same kind of schemes has been considered in [6] where the convergence in probability to the solution of the continuous equation is proved, in the case where the noise is a space-time potential and is white in time. The order of convergence of a Crank-Nicolson semi-discrete scheme as also been studied in [16], for the Manakov-PMD system, a system of NLS equations with a threedimensional time white noise appearing in the equation as a coefficient of the gradient of the solution. This model also appears in nonlinear fiber optics, and the (strong) order of convergence in time was then found to be at least $1 / 2$. The order of convergence of a splitting scheme was studied in [18] for equation (1.1), except that the nonlinear term was replaced by a Lipschitz function. Asymptotic preserving properties of the scheme have also been proved $([13])$. The order of convergence was also found to be at least $1 / 2$, although it is expected to be equal to one in this case, since the noise is one-dimensional. We indeed prove in Section 2 that the strong order in probability for the time semi-discrete Crank-Nicolson scheme is at least 1. 
Note that the spatial discretization is very easy to analyse. Indeed, from our argument it is clear that it suffices to study (1.1) with a Lipschitz nonlinearity. Then, classical arguments show that the spatial discretization error is the same as in the deterministic case.

We now give some notations. We will denote by $\mathcal{F}_{x}$ the spatial Fourier transform in $\mathbb{R}^{d}$ and $\hat{u}$ denotes also the (space) Fourier transform of the (complex-valued) function $u$. For any integer (or even real) number $s$, we denote by $H^{s}\left(\mathbb{R}^{d}\right)$ the Sobolev space of complex-valued distributions $u$ on $\mathbb{R}^{d}$ satisfying $(I-\Delta)^{s / 2} u \in L^{2}\left(\mathbb{R}^{d}\right)$ and by $\|\cdot\|_{H^{s}}$ its norm. This is a real Hilbert space, which thanks to the Plancherel Theorem is endowed with the inner product

$$
(u, v)_{s} \equiv \mathcal{R} e \int_{\mathbb{R}^{d}}\left(1+|\xi|^{2}\right)^{s} \hat{u}(\xi) \overline{\hat{v}}(\xi) d \xi
$$

Note that here we identify $\mathbb{C}$ with $\mathbb{R}^{2}$, and we will say that a function $f$ defined on $H^{s}\left(\mathbb{R}^{d}\right)$ is in $C_{b}^{2}\left(H^{s}\right)$ if $f$ is of class $C^{2}$ on $H^{s}\left(\mathbb{R}^{d} ; \mathbb{R}\right) \times H^{s}\left(\mathbb{R}^{d} ; \mathbb{R}\right)$ and has bounded derivatives, i.e. there is a constant $C>0$ such that for any $u, v, w \in H^{s}\left(\mathbb{R}^{d}\right)$

$$
\|D f(u) \cdot v\|_{H^{s}} \leq C\|v\|_{H^{s}}
$$

and

$$
\left\|D^{2} f(u)(v, w)\right\|_{H^{s}} \leq C\|v\|_{H^{s}}\|w\|_{H^{s}}
$$

where $D^{2} f(u)$ denotes the second order derivative of $f$ at $u$, and is a bilinear operator from $H^{s}\left(\mathbb{R}^{d}\right) \times H^{s}\left(\mathbb{R}^{d}\right)$ into $H^{s}\left(\mathbb{R}^{d}\right)$. With obvious modifications in the definition, we will also consider functions $g$ which belong to $C_{b}^{2}\left(H^{s} \times H^{s}\right)$. We will in that case denote $D_{1} g$ (resp. $D_{2} g$ ) the partial differential with respect to the first (resp. second) argument of $g$.

\section{Numerical analysis of the CRANK-Nicolson SCheme}

2.1. Preliminaries and main result. We consider in this section equation (1.1) in the cases where $\sigma$ is an integer, which makes the power term $|u|^{2 \sigma} u$ regular in $u$ and allows us to differentiate it as many times as we need. Moreover, we will assume that the existence and uniqueness of a global solution is known, which is the case e.g. if $\sigma=1$ or $\sigma=2$, and the space dimension $d$ is equal to one (see Proposition 2.1 below). We recall (see $[9,18])$ that equation (1.1) may also be written in the mild form

$$
u(t)=S(t, 0) u_{0}+i \int_{0}^{t} S(t, s)\left(|u|^{2 \sigma} u(s)\right) d s
$$

where the random propagator $S(t, s)$ assigns to any $\mathcal{F}_{s}$-measurable $L^{2}$-valued random variable $u_{s}$ the solution at time $t>s$ of the linear equation

$$
\left\{\begin{array}{l}
i d u+\frac{i}{2} \Delta^{2} u d t+\Delta u d \beta=0, \\
u(s)=u_{s}
\end{array}\right.
$$


Note that $S(t, s)$ has an explicit expression in Fourier variables given by

$$
\mathcal{F}\left(S(t, s) u_{s}\right)(\xi)=e^{-i|\xi|^{2}(\beta(t)-\beta(s))} \hat{u}_{s}(\xi), t \geq s, \xi \in \mathbb{R}^{d},
$$

(see [9]) and this allows to generalize the definition of $S(t, s) u$ to non necessarily $\mathcal{F}_{s^{-}}$ measurable random functions $u$.

Our assumption on global existence and uniqueness result is known to be true in some cases: it has been proved for $s=1$, in [9] in the case $\sigma=1$ and in [11] for the case $\sigma=2$. The extension of the result to a general Sobolev space $H^{s}\left(\mathbb{R}^{d}\right)$ with $s \geq 1$, i.e. the fact that the solution $u$ has trajectories a.s. in $C\left(\mathbb{R}^{+} ; H^{s}(\mathbb{R})\right)$ if $u_{0} \in H^{s}(\mathbb{R})$ is an immediate consequence of the embedding $H^{1} \subset L^{\infty}(\mathbb{R})$ and of the isometry of $S(t, s)$ in $H^{s}(\mathbb{R})$ for any $s$. We recall these results for completeness.

Proposition 2.1. Let $d=1, \sigma=1$ or 2 , and $u_{0} \in H^{s}(\mathbb{R})$ with $s \geq 1$. Then there exists a unique adapted solution of equation (2.1) with paths a.s. in $C\left(\mathbb{R}^{+} ; H^{s}(\mathbb{R})\right)$.

We expect that global existence and uniqueness can be proved for $\sigma<4 / d$ - see the numerical simulations of Section 3 - so that our result would also hold true with $\sigma=1$ for $d=2,3$.

Our aim in this section is to study the following time discretization of equation (1.1), where $\delta t>0$ denotes the time step :

$$
i \frac{u_{n+1}-u_{n}}{\delta t}+\frac{\chi_{n}}{\sqrt{\delta t}} \Delta u_{n+1 / 2}+g\left(u_{n}, u_{n+1}\right)=0
$$

where the function $g$ is an approximation of the nonlinear term $|u|^{2 \sigma} u$ given by

$$
\begin{aligned}
g(u, v) & =\int_{0}^{1}\left(\theta|u|^{2}+(1-\theta)|v|^{2}\right)^{\sigma} d \theta\left(\frac{u+v}{2}\right) \\
& =\frac{1}{\sigma+1}\left(\frac{|u|^{2 \sigma+2}-|v|^{2 \sigma+2}}{|u|^{2}-|v|^{2}}\right)\left(\frac{u+v}{2}\right) .
\end{aligned}
$$

Here $u_{n+1 / 2}=\frac{1}{2}\left(u_{n}+u_{n+1}\right)$. Moreover, the random variables $\chi_{n}$ are defined by $\chi_{n}=$ $\frac{\beta((n+1) \delta t)-\beta(n \delta t)}{\sqrt{\delta t}}$. Hence the family $\left(\chi_{n}\right)_{n} \in \mathbb{N}$ is an independent family of normal random variables. With these definitions, $u_{n}$ is then an approximation of $u\left(t_{n}\right)$, where $t_{n}=n \delta t$ and $u$ is the solution of equation (1.1).

The mid-point discretization of the noise term is natural to approximate a Stratonovich product. This scheme is a generalization to equation (1.1) of the classical Crank-Nicolson scheme for the deterministic NLS equation, which is known to be conservative (in the deterministic case) for the $L^{2}$-norm and the Hamiltonian (see e.g. [12] or [20]). For the stochastic NLS equation (1.1) with a power nonlinearity, the scheme is conservative for the $L^{2}$-norm (see Section 3).

Another possibility would have been to consider an explicit discretization of the noise term in the Itô formulation (1.2) of the equation. However, such a discretization would 
not lead to a conservative scheme. On the other hand, the scheme (2.3) may be written as

$$
i \frac{u_{n+1}-u_{n}}{\delta t}+\frac{\chi_{n}}{\sqrt{\delta t}} \Delta u_{n}+\frac{1}{2} \frac{\chi_{n}}{\sqrt{\delta t}} \Delta\left(u_{n+1}-u_{n}\right)+g\left(u_{n}, u_{n+1}\right)=0
$$

from which we deduce (assuming that each term above is bounded and that $g$ is sufficiently regular and bounded)

$$
\frac{\Delta\left(u_{n+1}-u_{n}\right)}{\sqrt{\delta t}}=i \chi_{n} \Delta^{2} u_{n}+O(\sqrt{\delta t})
$$

plugging this expression into the preceding equation, we obtain up to the $O(\sqrt{\delta t})$ term the new formulation for the scheme $(2.3)$

$$
i \frac{u_{n+1}-u_{n}}{\delta t}+\frac{\chi_{n}}{\sqrt{\delta t}} \Delta u_{n}+\frac{i}{2} \chi_{n}^{2} \Delta^{2} u_{n}+g\left(u_{n}, u_{n+1}\right)=0,
$$

which is exactly the Milstein scheme for the Itô equation (1.2) (see [19]). Hence we may expect that the scheme has an order of convergence in time equal to one. This is indeed the main result of this section (see Theorem 2.3 below).

We now introduce some notations. For a fixed $n \in \mathbb{N}$, we denote by $S_{n, \delta t}$ the linear operator

$$
S_{n, \delta t}=\left(I-i \frac{\sqrt{\delta t}}{2} \chi_{n} \Delta\right)^{-1}\left(I+i \frac{\sqrt{\delta t}}{2} \chi_{n} \Delta\right)
$$

This operator is a Fourier multiplier, it is a bounded linear operator on $L^{2}\left(\mathbb{R}^{d}\right)$ satisfying

$$
\mathcal{F}\left(S_{n, \delta t} u\right)(\xi)=\frac{1-i \frac{\sqrt{\delta t}}{2} \chi_{n}|\xi|^{2}}{1+i \frac{\sqrt{\delta t}}{2} \chi_{n}|\xi|^{2}} \hat{u}(\xi)
$$

for any $u \in L^{2}\left(\mathbb{R}^{d}\right)$. We also denote by $T_{n, \delta t}$ the linear operator $T_{n, \delta t}=\left(I-i \frac{\sqrt{\delta t}}{2} \chi_{n} \Delta\right)^{-1}$, which is also a linear bounded operator in $L^{2}\left(\mathbb{R}^{d}\right)$. Then the scheme $(2.3)$ may be written as

$$
u_{n+1}=S_{n, \delta t} u_{n}+i(\delta t) T_{n, \delta t} g\left(u_{n}, u_{n+1}\right) .
$$

At that point, we should mention that because $g$ is only a locally Lipschitz function on $\mathbb{C}^{2}$ - hence the Nemytskii operator $(u, v) \mapsto g(u, v)$ is locally Lipschitz in $H^{s}\left(\mathbb{R}^{d}\right) \times H^{s}\left(\mathbb{R}^{d}\right)$ for any $s>d / 2$ - the existence, for a given $u_{n}$ in $H^{s}\left(\mathbb{R}^{d}\right)$, of $u_{n+1} \in H^{s}\left(\mathbb{R}^{d}\right)$ satisfying (2.5) is not obvious ; moreover, $u_{n+1}$ will not be unique in general. In order to define the solution in a simple way, we thus introduce a cut-off in the nonlinear part of the scheme.

Let $\theta \in C^{\infty}\left(\mathbb{R}^{+}\right)$with $\theta \geq 0, \operatorname{supp} \theta \subset[0,2]$ and $\theta \equiv 1$ on $[0,1]$; we then set $\theta_{k}(x)=\theta\left(\frac{x}{k}\right)$, for $x \geq 0$ and $k \in \mathbb{N}$. Now, in all what follows, the integer $s>d / 2$ is fixed, and we set for $k \in \mathbb{N}$ and $u, v \in H^{s}\left(\mathbb{R}^{d}\right)$ :

$$
g_{k}(u, v)=\frac{1}{2 \sigma+2} \theta_{k}\left(|u|_{H^{s}}^{2}\right) \theta_{k}\left(|v|_{H^{s}}^{2}\right)\left(\frac{|u|^{2 \sigma+2}-|v|^{2 \sigma+2}}{|u|^{2}-|v|^{2}}\right)\left(\frac{u+v}{2}\right) .
$$


It is clear that $g_{k}$ is a Lipschitz function on $H^{s}\left(\mathbb{R}^{d}\right)$ and satisfies : for any $u_{1}, u_{2}, v_{1}, v_{2} \in$ $H^{s}\left(\mathbb{R}^{d}\right)$,

$$
\left|g_{k}\left(u_{1}, v_{1}\right)-g_{k}\left(u_{2}, v_{2}\right)\right|_{H^{s}} \leq C(\sigma, k)\left(\left|u_{1}-u_{2}\right|_{H^{s}}+\left|v_{1}-v_{2}\right|_{H^{s}}\right) .
$$

We then replace the scheme $(2.5)$ by

$$
u_{n+1}^{k}=S_{n, \delta t} u_{n}^{k}+i(\delta t) T_{n, \delta t} g_{k}\left(u_{n}^{k}, u_{n+1}^{k}\right) .
$$

The next proposition may be proved as Proposition 3.1 in [16], using the integral formulation of (2.7) and a fixed point argument in the subspace of $L^{\infty}\left(0, T ; H^{s}\left(\mathbb{R}^{d}\right)\right)$ consisting of functions which are constant on each interval $[n \delta t,(n+1) \delta t], 0 \leq n \leq N-1$, with $\delta t=T / N$, provided that this latter is less than $1 / C(\sigma, k)$, where $C(\sigma, k)$ the Lipschitz constant of $g_{k}$, together with the fact that the operators $S_{n, \delta t}$ and $T_{n, \delta t}$ are bounded in $H^{s}\left(\mathbb{R}^{d}\right)$ with bound less than one. For general $\delta t$, one obtains the existence of a discrete solution by using a measurable selection theorem (see e.g. [8, 16]), but the solution is not unique in general.

Proposition 2.2. Let $T>0$ and $\delta t=T / N>0$ be fixed. Let $u_{0} \in H^{s}\left(\mathbb{R}^{d}\right)$ with $s>d / 2$; then for any $k \in \mathbb{N}^{*}$, there is an adapted discrete solution $u_{\delta t}^{k}=\left(u_{n}^{k}\right)_{n=0, \ldots, N}$ satisfying (2.7) for $n \leq N-1$, which belongs a.s. to $L^{\infty}\left(0, T ; H^{s}\left(\mathbb{R}^{d}\right)\right)$, and the solution $u_{\delta t}^{k}$ is unique if $\delta t$ is sufficiently small, depending on $k$. Moreover, for any $n \leq N$, the $L^{2}$ norm is preserved that is $\left\|u_{n}^{k}\right\|_{L^{2}}=\left\|u_{0}\right\|_{L^{2}}$.

Now, we define the stopping time

$$
\tau_{\delta t}^{k}=\inf \left\{n \delta t,\left\|u_{n-1}^{k}\right\|_{H^{s}} \geq k, \text { or }\left\|u_{n}^{k}\right\|_{H^{s}} \geq k\right\},
$$

which is nondecreasing with $k$. It is clear that $\left(u_{n}^{k}\right)_{n=0, \ldots, n_{0}-1}$ satisfies the scheme (2.5) as long as $n_{0} \delta t \leq \tau_{\delta t}^{k}$. Moreover the discrete solution given by Proposition 2.2 can be constructed in such a way that $u_{n}^{k+1}=u_{n}^{k}$ for $n \delta t \leq \tau_{\delta t}^{k}$.

Finally, we set for a fixed $\delta t=T / N>0: u_{n}^{\delta t}=u_{n}^{k}$ if $n \delta t \leq \tau_{\delta t}^{k}$. This allows to define the discrete solution of the scheme (2.3) up to the stopping time $\tau_{\delta t}^{\infty} \equiv \lim _{k \rightarrow \infty} \tau_{\delta t}^{k} \wedge T$. For $t \geq \tau_{\delta t}^{\infty}$, we just set $u_{n}^{\delta t}=u_{\infty}$, where $u_{\infty}$ is any arbitrary element in $H^{s}\left(\mathbb{R}^{d}\right)$. We are now in position to state our main result, which says that the scheme (2.3) has strong order one in probability.

Theorem 2.3. Let $T>0$; assume $u_{0} \in H^{s}\left(\mathbb{R}^{d}\right), s>d / 2$, is such that equation (2.1) has a unique adapted solution $u$ with paths a.s. in $C\left([0, T]: H^{s}\left(\mathbb{R}^{d}\right)\right)$. For $\delta t=T / N$, with $N \in \mathbb{N}^{*}$, let $t_{n}=n \delta t, n=0, \ldots, N$; then for any $n \leq N$,

$$
\lim _{C \rightarrow \infty} \mathbb{P}\left(\left\|u\left(t_{n}\right)-u_{n}^{\delta t}\right\|_{H^{s}} \geq C \delta t\right)=0
$$

uniformly with respect to $\delta t$.

Remark 2.4. We have assumed for simplicity that the solution of the continuous equation (2.1) was defined on a deterministic time interval, but the result would also be true if the solution was only locally defined in time, on a random time interval which is a stopping time (see $[8,16])$. In particular the result would be true for $s>d / 2$ in $H^{s}\left(\mathbb{R}^{d}\right)$, 
or in $H^{s}\left(\mathbb{T}^{d}\right)$, where $\mathbb{T}=\mathbb{R} / 2 \pi \mathbb{Z}$, using in all what follows Fourier series instead of the continuous Fourier transform

Remark 2.5. We actually expect that a stronger result holds under the assumptions of Theorem 2.3, namely we expect that

$$
\lim _{C \rightarrow \infty} \mathbb{P}\left(\sup _{n \delta t \leq T}\left\|u\left(t_{n}\right)-u_{n}^{\delta t}\right\|_{H^{s}} \geq C \delta t\right)=0,
$$

uniformly with respect to $\delta t$. This would e.g. follow from the arguments in [8] if we were able to prove a mean square order one, for the truncated scheme 2.7, in the $L^{\infty}\left(0, T ; H^{s}\left(\mathbb{R}^{d}\right)\right)$ norm (see Proposition 2.6) below. In turn, this latter result would follow from the arguments in [14], Chapter 5, if we were able to prove that the scheme 2.7 approximates the continuous equation at order one in $L^{p}(\Omega)$ for any $p \geq 1$. However, the scheme being implicit in the noise, we cannot use the Itô Formula, and obtaining an order in $L^{p}(\Omega)$ seems to require very tedious computations, while our argument to get Theorem 2.3 from the mean square result for the truncated scheme given by Proposition 2.6 below is extremely simple.

In order to prove Theorem 2.3 we will thus first prove that the scheme defined by (2.7) gives an approximation of the corresponding continuous equation of order one in mean square. This will be the object of the next subsection.

2.2. Mean square order for the truncated scheme. In this subsection, we consider the scheme (2.7), where the integer $k \in \mathbb{N}$ is fixed, and we prove that its solution $u_{\delta t}^{k}$ given by Proposition 2.2 converges in mean square at order one to the solution of the continuous equation :

$$
u(t)=S(t, 0) u_{0}+i \int_{0}^{t} S(t, s) f_{k}(u(s)) d s
$$

where

$$
f_{k}(u)=\theta_{k}\left(\|u\|_{H^{s}}^{2}\right)|u|^{2 \sigma} u \text {. }
$$

Note that, for a fixed $k \in \mathbb{N}$, since $s>d / 2, f_{k}$ is a Lipschitz function on $H^{s}\left(\mathbb{R}^{d}\right)$, and the existence of a unique global adapted solution of $(2.9)$ in $L^{\infty}\left(\Omega ; C\left([0, T] ; H^{s}\left(\mathbb{R}^{d}\right)\right)\right)$ is immediate, if $u_{0} \in H^{s}\left(\mathbb{R}^{d}\right)$. Moreover, using an induction argument and the fact that $S(t, s)$ is an isometry in $H^{\alpha}\left(\mathbb{R}^{d}\right)$ for any $\alpha$, it is not difficult to see that if in addition $u_{0} \in H^{s+6}\left(\mathbb{R}^{d}\right)$, then $u_{k}$ has paths a.s. in $H^{s+6}\left(\mathbb{R}^{d}\right)$, and there is a deterministic constant $C=C\left(T, k,\left\|u_{0}\right\|_{H^{s}}\right)$ such that

$$
\left\|u_{k}\right\|_{C\left([0, T] ; H^{s+6}\right)} \leq C, \quad \text { a.s. }
$$

Proposition 2.6. Let $T>0$ and $u_{0} \in H^{s+6}\left(\mathbb{R}^{d}\right)$. Let $k \in \mathbb{N}$ be fixed, $u_{k}$ be the unique adapted solution of (2.9) with paths a.s. in $C\left([0, T] ; H^{s+6}\left(\mathbb{R}^{d}\right)\right)$, and $u_{\delta t}^{k}=$ $\left(u_{n}^{k}\right)_{n=0, . ., N}$ with $\delta t=T / N$ be an adapted discrete solution of (2.7) given by Proposition 2.2, where $g_{k}$ is defined by (2.6). Then there is a constant $C$ which may depend on 
$T$ and $\sup _{t \in[0, T]} \mathbb{E}\left(\|u(t)\|_{H^{s+6}}^{4}\right)$ such that for any $\delta t=T / N$,

$$
\sup _{n \delta t \leq T} \mathbb{E}\left(\left\|u_{n}^{k}-u_{k}\left(t_{n}\right)\right\|_{H^{s}}^{2}\right) \leq C(\delta t)^{2}
$$

where $t_{n}=n \delta t$.

Proof of Proposition 2.6. In all the proof, since the index $k$ refereeing to the cut-off is fixed, we ignore it and simply denote $u_{n}^{k}$ by $u_{n}, u_{k}$ by $u$, and so on. Moreover, all the constants appearing in the subsection may depend on $k$, even though this is not always explicitly stated. We then denote by $e_{n}$ the error $e_{n}=u_{n}-u\left(t_{n}\right)$ where we recall that $t_{n}=n \delta t$. In order to prove Proposition 2.6, we first prove that there are positive constants $\varepsilon, K_{1}$ and $K_{2}$, that possibly depend on $\sup _{t \in[0, T]} \mathbb{E}\left(\|u(t)\|_{H^{s+6}}^{4}\right)$ such that

$$
\mathbb{E}\left(\left\|e_{n+1}\right\|_{H^{s}}^{2}\right) \leq\left(1+(\delta t) K_{1}\right) \mathbb{E}\left(\left\|e_{n}\right\|_{H^{s}}^{2}\right)+(\delta t)^{3} K_{2}
$$

for all $\delta t \leq \varepsilon$ and all $n \in \mathbb{N}$ such that $n \delta t \leq T$.

Using the mild form of the scheme (2.7), and the expression

$$
u\left(t_{n+1}\right)=S\left(t_{n+1}, t_{n}\right) u\left(t_{n}\right)+i \int_{t_{n}}^{t_{n+1}} S\left(t_{n+1}, s\right) f_{k}(u(s)) d s .
$$

which is deduced from (2.9), one may express $e_{n+1}$ in terms of $e_{n}$ as follows:

$$
e_{n+1}=\sum_{j=1}^{5} e_{n, \delta t, j}
$$

where

$$
\begin{gathered}
e_{n, \delta t, 1}=S_{n, \delta t} e_{n}, \\
e_{n, \delta t, 2}=\left(S_{n, \delta t}-S\left(t_{n+1}, t_{n}\right)\right) u\left(t_{n}\right), \\
e_{n, \delta t, 3}=i \int_{t_{n}}^{t_{n+1}} S\left(t_{n+1}, s\right)\left(g_{k}\left(u\left(t_{n}\right), u\left(t_{n+1}\right)\right)-f_{k}(u(s))\right) d s \\
e_{n, \delta t, 4}=i\left((\delta t) T_{n, \delta t}-\int_{t_{n}}^{t_{n+1}} S\left(t_{n+1}, s\right) d s\right) g_{k}\left(u\left(t_{n}\right), u\left(t_{n+1}\right)\right),
\end{gathered}
$$

and

$$
e_{n, \delta t, 5}=i(\delta t) T_{n, \delta t}\left(g_{k}\left(u_{n}, u_{n+1}\right)-g_{k}\left(u\left(t_{n}\right), u\left(t_{n+1}\right)\right)\right) .
$$

We will estimate separately the contribution of each of the above terms to the left hand side of (2.11). Indeed, we may write using (2.13) :

$$
\mathbb{E}\left(\left\|e_{n+1}\right\|_{H^{s}}^{2}\right)=\sum_{j=1}^{5} \mathbb{E}\left(\left\|e_{n, \delta t, j}\right\|_{H^{s}}^{2}\right)+2 \sum_{1 \leq i<j \leq 5} \mathbb{E}\left(e_{n, \delta t, i}, e_{n, \delta t, j}\right)_{s}
$$

where we recall that $(., .)_{s}$ denotes the inner product in $H^{s}\left(\mathbb{R}^{d}\right)$. 
First, it is obvious from its expression in Fourier space (we recall that $\chi_{n}$ is a real valued Gaussian random variable) that the operator $S_{n, \delta t}$ is a unitary operator on $H^{s}\left(\mathbb{R}^{d}\right)$. It follows

$$
\mathbb{E}\left(\left\|e_{n, \delta t, 1}\right\|_{H^{s}}^{2}\right)=\mathbb{E}\left(\left\|e_{n}\right\|_{H^{s}}^{2}\right) .
$$

The second term, which corresponds to the approximation of the linear equation, is estimated thanks to the next lemma.

Lemma 2.7. There is a constant $C$ such that for any random function $v \in L^{4}\left(\Omega ; H^{s+6}\left(\mathbb{R}^{d}\right)\right)$,

$$
\mathbb{E}\left(\left\|S\left(t_{n+1}, t_{n}\right) v-S_{n, \delta t} v\right\|_{H^{s}}^{2}\right) \leq C(\delta t)^{3}\left(\mathbb{E}\left(\|v\|_{H^{s+6}}^{4}\right)\right)^{1 / 2} .
$$

If moreover, $v$ is $\mathcal{F}_{t_{n}}$-measurable, then

$$
\mathbb{E}\left(\left\|S\left(t_{n+1}, t_{n}\right) v-S_{n, \delta t} v\right\|_{H^{s}}^{2}\right) \leq C(\delta t)^{3} \mathbb{E}\left(\|v\|_{H^{s+6}}^{2}\right) .
$$

Applying Lemma 2.7 with $v=u\left(t_{n}\right)$ immediately leads to the estimate

$$
\mathbb{E}\left(\left\|e_{n, \delta t, 2}\right\|_{H^{s}}^{2}\right) \leq C(\delta t)^{3} \sup _{t \in[0, T]} \mathbb{E}\left(\|u(t)\|_{H^{s+6}}^{2}\right),
$$

for any $n$ such that $n \delta t \leq T$.

Proof of Lemma 2.7. Thanks to the expression of the operator $S_{n, \delta t}$ as a Fourier multiplier, we may write, for any $\xi \in \mathbb{R}^{d}$ :

$$
\mathcal{F}\left(S_{n, \delta t} v\right)(\xi)=\frac{1-i \frac{\sqrt{\delta t}}{2} \chi_{n}|\xi|^{2}}{1+i \frac{\sqrt{\delta t}}{2} \chi_{n}|\xi|^{2}} \hat{v}(\xi)=e^{-2 i \arctan \left(\frac{\sqrt{\delta t}}{2}|\xi|^{2} \chi_{n}\right)} \hat{v}(\xi) .
$$

Hence the Parseval Theorem implies that for any random function $v \in L^{2}\left(\Omega ; H^{s+6}\left(\mathbb{R}^{d}\right)\right)$,

$$
\mathbb{E}\left(\left\|S\left(t_{n+1}, t_{n}\right) v-S_{n, \delta t} v\right\|_{H^{s}}^{2}\right)=\int_{\mathbb{R}^{d}} \mathbb{E}\left(\left|e^{-i \sqrt{\delta t}|\xi|^{2} \chi_{n}}-e^{-2 i \arctan \left(\frac{\sqrt{\delta t}}{2}|\xi|^{2} \chi_{n}\right)}\right|^{2}|\hat{v}(\xi)|^{2}\right)\left(1+|\xi|^{2}\right)^{s} d \xi
$$

Now, since $\left|1-e^{i x}\right|^{2} \leq C|x|^{2}$ for any real $x$, and $|\arctan y-y| \leq C|y|^{3}$ for any $y \in \mathbb{R}$, where the constants $C$ are independent of $x$ and $y$, the previous equality implies

$$
\mathbb{E}\left(\left\|S\left(t_{n+1}, t_{n}\right) v-S_{n, \delta t} v\right\|_{H^{s}}^{2}\right) \leq C(\delta t)^{3} \mathbb{E}\left(\chi_{n}^{6} \int_{\mathbb{R}^{d}}\left(1+|\xi|^{2}\right)^{s}|\xi|^{12}|\hat{v}(\xi)|^{2} d \xi\right) .
$$

If moreover, $v$ is independent of $\chi_{n}$, then the right hand side above is bounded by

$$
C(\delta t)^{3} \mathbb{E}\left(\chi_{n}^{6}\right) \mathbb{E}\left(\|v\|_{H^{s+6}}^{2}\right)
$$

and we may conclude in the general case using Cauchy-Schwarz inequality.

We now turn to the estimate of $\mathbb{E}\left(\left\|e_{n, \delta t, 3}\right\|_{L^{2}}^{2}\right)$. For this purpose, we will use a Taylor expansion of the function (in fact the Nemytskii operator) $g_{k}$. Indeed, we recall that since $\sigma$ is an integer, there exists a real valued polynomial function $p_{\sigma}\left(x_{1}, x_{2}\right)$ such that, for all $u, v \in H^{s}\left(\mathbb{R}^{d}\right)$ and all $x \in \mathbb{R}^{d}$,

$$
g_{k}(u, v)(x)=\theta_{k}\left(|u|_{H^{s}}^{2}\right) \theta_{k}\left(|v|_{H^{s}}^{2}\right) p_{\sigma}\left(|u(x)|^{2},|v(x)|^{2}\right) .
$$


It easily follows that $g_{k}$ is differentiable on $H^{s}\left(\mathbb{R}^{d}\right) \times H^{s}\left(\mathbb{R}^{d}\right)$, and for any $(u, v) \in H^{s} \times H^{s}$, and any $w \in H^{s}$, the function $D_{u} g_{k}(u, v) . w$ belongs to $H^{s}\left(\mathbb{R}^{d}\right)$ and $\forall x \in \mathbb{R}^{d}$,

$$
\begin{aligned}
\left(D_{u} g_{k}(u, v) . w\right)(x)= & \theta_{k}\left(|u|_{H^{s}}^{2}\right) \theta_{k}\left(|v|_{H^{s}}^{2}\right) \partial_{x_{1}} p_{\sigma}\left(|u(x)|^{2},|v(x)|^{2}\right) 2 \mathcal{R} e(\bar{u}(x) w(x)) \\
& +2 \theta_{k}^{\prime}\left(|u|_{H^{s}}^{2}\right) \theta_{k}\left(|v|_{H^{s}}^{2}\right)(u, w)_{s} p_{\sigma}\left(|u(x)|^{2},|v(x)|^{2}\right) .
\end{aligned}
$$

A similar expression holds for $D_{v} g_{k}(u, v) . w$, and we deduce that there is a constant $C(k)$, which only depends on $k$, such that for any $(u, v) \in H^{s} \times H^{s}$, and any $\left(w_{1}, w_{2}\right) \in$ $H^{s} \times H^{s}$,

$$
\left\|D g_{k}(u, v) \cdot\left(w_{1}, w_{2}\right)\right\|_{H^{s}} \leq C(k)\left(\left\|w_{1}\right\|_{H^{s}}+\left\|w_{2}\right\|_{H^{s}}\right),
$$

in other words, $g_{k} \in C_{b}^{1}\left(H^{s} \times H^{s}\right)$. In the same way, it may be checked that $g_{k} \in$ $C_{b}^{2}\left(H^{s} \times H^{s}\right)$, i.e. for any $(u, v) \in H^{s} \times H^{s}$, and any $\left(w_{1}, w_{2}\right) \in H^{s} \times H^{s}$,

$$
\left\|D^{2} g_{k}(u, v) \cdot\left(w_{1}, w_{2}\right)^{2}\right\|_{H^{s}} \leq C(k)\left(\left\|w_{1}\right\|_{H^{s}}^{2}+\left\|w_{2}\right\|_{H^{s}}^{2}\right) \text {. }
$$

Then, by Taylor expansion, we may write, for any $s \in\left(t_{n}, t_{n+1}\right)$,

$$
\begin{aligned}
& f_{k}(u(s))=g_{k}(u(s), u(s)) \\
& =g_{k}\left(u\left(t_{n}\right), u\left(t_{n+1}\right)\right)+\int_{0}^{1} D g_{k}\left(u\left(t_{n}\right)+\theta \mathcal{E}_{n}(s), u\left(t_{n+1}\right)+\theta \mathcal{E}_{n+1}(s)\right)\left(\mathcal{E}_{n}(s), \mathcal{E}_{n+1}(s)\right) d \theta
\end{aligned}
$$

where $\mathcal{E}_{n}(s)=u(s)-u\left(t_{n}\right)$. Using (2.23) above in the expression of $e_{n, \delta t, 3}$ given by (2.16), it follows by Cauchy-Schwarz inequality, since $g_{k}$ has bounded first derivatives, that

$$
\begin{aligned}
& \mathbb{E}\left(\left\|e_{n, \delta t, 3}\right\|_{H^{s}}^{2}\right) \\
\leq & C(\delta t) \int_{t_{n}}^{t_{n+1}} \int_{0}^{1} \mathbb{E}\left\|D g_{k}\left(u\left(t_{n}\right)+\theta \mathcal{E}_{n}(s), u\left(t_{n+1}\right)+\theta \mathcal{E}_{n+1}(s)\right)\left(\mathcal{E}_{n}(s), \mathcal{E}_{n+1}(s)\right)\right\|_{H^{s}}^{2} d \theta d s \\
\leq & C(\delta t) \int_{t_{n}}^{t_{n+1}} \mathbb{E}\left(\left\|\mathcal{E}_{n}(s)\right\|_{H^{s}}^{2}+\left\|\mathcal{E}_{n+1}(s)\right\|_{H^{s}}^{2}\right) d s .
\end{aligned}
$$

In order to estimate the right hand side above, $\mathcal{E}_{n}(s)$ is written using again the mild form (2.9) of the continuous equation as :

$$
\mathcal{E}_{n}(s)=u(s)-u\left(t_{n}\right)=S\left(s, t_{n}\right) u\left(t_{n}\right)-u\left(t_{n}\right)+i \int_{t_{n}}^{s} S(s, \sigma) f_{k}(u(\sigma)) d \sigma
$$

and we use the following lemma.

Lemma 2.8. There is a constant $C$ such that for any random function $v \in L^{4}\left(\Omega ; H^{s+2}\left(\mathbb{R}^{d}\right)\right)$, and for any $s \in\left(t_{n}, t_{n+1}\right)$,

$$
\mathbb{E}\left(\left\|S\left(s, t_{n}\right) v-v\right\|_{H^{s}}^{2}\right) \leq C(\delta t)\left(\mathbb{E}\left(\|v\|_{H^{s+2}}^{4}\right)\right)^{1 / 2} .
$$

If moreover, $v$ is $\mathcal{F}_{t_{n}}$-measurable, then

$$
\mathbb{E}\left(\left\|S\left(s, t_{n}\right) v-v\right\|_{H^{s}}^{2}\right) \leq C(\delta t) \mathbb{E}\left(\|v\|_{H^{s+2}}^{2}\right),
$$


and

$$
\mathbb{E}\left(\left\|S\left(s, t_{n}\right) v-v\right\|_{H^{s}}^{4}\right) \leq C(\delta t)^{2} \mathbb{E}\left(\|v\|_{H^{s+2}}^{4}\right) .
$$

Proof of Lemma 2.8. The first inequality requires the same arguments as the proof of Lemma 2.7 : the inequality $\left|e^{i x}-1\right|^{2} \leq C|x|^{2}$, which is true for any real $x$, implies, for any $v \in L^{4}\left(\Omega ; H^{s+2}\left(\mathbb{R}^{d}\right)\right)$, and for any $s \in\left(t_{n}, t_{n+1}\right)$ :

$$
\begin{aligned}
& \mathbb{E}\left(\left|\mathcal{F}_{x}\left(S\left(s, t_{n}\right) v-v\right)(\xi)\right|^{2}\right) \\
& =\mathbb{E}\left(\left|e^{-i\left(\beta(s)-\beta\left(t_{n}\right)\right)|\xi|^{2}}-1\right|^{2}|\hat{v}(\xi)|^{2}\right) \\
& \leq C \mathbb{E}\left(\left|\beta(s)-\beta\left(t_{n}\right)\right|^{2}|\xi|^{4}|\hat{v}(\xi)|^{2}\right) .
\end{aligned}
$$

Parseval Theorem and Cauchy-Schwarz inequality then easily lead to the first inequalities of Lemma 2.8. On the other hand, the above argument and Parseval Theorem imply, for any $\mathcal{F}_{t_{n}}$-measurable $v \in L^{4}\left(\Omega ; H^{s+2}\left(\mathbb{R}^{d}\right)\right)$,

$$
\begin{aligned}
& \mathbb{E}\left(\left\|S\left(s, t_{n}\right) v-v\right\|_{H^{s}}^{4}\right) \\
= & \mathbb{E} \int_{\mathbb{R}^{d}} \int_{\mathbb{R}^{d}}\left|e^{i\left(\beta(s)-\beta\left(t_{n}\right)\right)|\xi|^{2}}-1\right|^{2}\left|e^{i\left(\beta(s)-\beta\left(t_{n}\right)\right)|\eta|^{2}}-1\right|^{2}\left(1+|\xi|^{2}\right)^{s}|\hat{v}(\xi)|^{2}\left(1+|\eta|^{2}\right)^{s}|\hat{v}(\eta)|^{2} d \xi d \eta \\
\leq & C \int_{\mathbb{R}^{d}} \int_{\mathbb{R}^{d}}\left(1+|\xi|^{2}\right)^{s}\left(1+|\eta|^{2}\right)^{s}|\xi|^{4}|\eta|^{4} \mathbb{E}\left(\left|\beta(s)-\beta\left(t_{n}\right)\right|^{4}|\hat{v}(\xi)|^{2}|\hat{v}(\eta)|^{2}\right) d \xi d \eta
\end{aligned}
$$

which is bounded above by

$$
C(\delta t)^{2} \mathbb{E}\left[\int_{\mathbb{R}^{d}}\left(1+|\xi|^{2}\right)^{s+2}|\hat{v}(\xi)|^{2} d \xi\right]^{2}=C(\delta t)^{2} \mathbb{E}\left(\|v\|_{H^{s+2}}^{4}\right),
$$

using again the independence of $v$ and $\beta(s)-\beta\left(t_{n}\right)$. The third inequality of Lemma 2.8 follows.

Lemma $2.8,(2.25)$ and the fact that $f_{k}$ is Lipschitz in $H^{s}\left(\mathbb{R}^{d}\right)$ with $f_{k}(0)=0$ immediately imply the bound

$$
\mathbb{E}\left(\left\|\mathcal{E}_{n}(s)\right\|_{H^{s}}^{2}\right) \leq C(\delta t) \sup _{t \in[0, T]} \mathbb{E}\left(\|u(t)\|_{H^{s+2}}^{2}\right)
$$

for any $s \in\left(t_{n}, t_{n+1}\right)$. Modifying the above arguments in an obvious way, one may also prove that

$$
\mathbb{E}\left(\left\|\mathcal{E}_{n+1}(s)\right\|_{H^{s}}^{2}\right) \leq C(\delta t) \sup _{t \in[0, T]} \mathbb{E}\left(\|u(t)\|_{H^{s+2}}^{2}\right)
$$

for any $s \in\left(t_{n}, t_{n+1}\right)$. From (2.24), (2.27) and (2.28), we deduce

$$
\mathbb{E}\left(\left\|e_{n, \delta t, 3}\right\|_{H^{s}}^{2}\right) \leq C(\delta t)^{3} \sup _{t \in[0, T]} \mathbb{E}\left(\|u(t)\|_{H^{s+2}}^{2}\right) .
$$


Let us now consider $\mathbb{E}\left(\left\|e_{n, \delta t, 4}\right\|_{H^{s}}^{2}\right)$. The expression (2.17) allows to write

$$
\begin{aligned}
\mathbb{E}\left(\left\|e_{n, \delta t, 4}\right\|_{H^{s}}^{2}\right) \leq & 2 \mathbb{E}\left(\left\|\int_{t_{n}}^{t_{n+1}}\left(I-S\left(t_{n+1}, s\right)\right) g_{k}\left(u\left(t_{n}\right), u\left(t_{n+1}\right)\right) d s\right\|_{H^{s}}^{2}\right) \\
& +2(\delta t)^{2} \mathbb{E}\left(\left\|\left(I-T_{n, \delta t}\right) g_{k}\left(u\left(t_{n}\right), u\left(t_{n+1}\right)\right)\right\|_{H^{s}}^{2} d s\right. \\
= & I+I I .
\end{aligned}
$$

The first term on the right hand side above is bounded thanks to the previous arguments : using Cauchy-Schwarz inequality,

$$
I \leq C(\delta t) \mathbb{E} \int_{t_{n}}^{t_{n+1}}\left\|\left(I-S\left(t_{n+1}, s\right)\right) g_{k}\left(u\left(t_{n}\right), u\left(t_{n+1}\right)\right)\right\|_{H^{s}}^{2} d s
$$

and by Lemma 2.8, and the fact that $g_{k}$ is Lipschitz in $H^{s+2}\left(\mathbb{R}^{d}\right) \times H^{s+2}\left(\mathbb{R}^{d}\right)$, as follows from (2.22) and the fact that $s$ is a positive integer,

$$
I \leq C(\delta t)^{3} \sup _{t \in[0, T]}\left(\mathbb{E}\left(\|u(t)\|_{H^{s+2}}^{4}\right)^{1 / 2} .\right.
$$

In order to estimate $I I$, we again use Parseval identity, and noticing that for any $\xi \in \mathbb{R}^{d}$,

$$
\left|\frac{1}{1+\frac{i \sqrt{\delta t}}{2} \chi_{n}|\xi|^{2}}-1\right|^{2} \leq \frac{\delta t}{4} \chi_{n}^{2}|\xi|^{4}
$$

we obtain, again with the use of Cauchy-Schwarz inequality and the fact that $g_{k}$ is Lipschitz in $H^{s+2}\left(\mathbb{R}^{d}\right) \times H^{s+2}\left(\mathbb{R}^{d}\right)$,

$$
\begin{aligned}
I I & \leq 2(\delta t)^{2} \mathbb{E} \int_{\mathbb{R}^{d}}\left|\frac{1}{1+\frac{i \sqrt{\delta t}}{2} \chi_{n}|\xi|^{2}}-1\right|^{2} \mid \mathcal{F}_{x}\left(\left.g\left(u\left(t_{n}\right), u\left(t_{n+1}\right)\right)(\xi)\right|^{2} d \xi\right. \\
& \leq C(\delta t)^{3}\left(\mathbb{E}\left(\chi_{n}^{4}\right)^{1 / 2}\right) \sup _{t \in[0, T]}\left[\mathbb{E}\left(\|u(t)\|_{H^{s+2}}^{4}\right)\right]^{1 / 2} .
\end{aligned}
$$

From (2.30) and these two estimates, we deduce

$$
\mathbb{E}\left(\left\|e_{n, \delta t, 4}\right\|_{H^{s}}^{2}\right) \leq C(\delta t)^{3}\left[1+\sup _{t \in[0, T]} \mathbb{E}\left(\|u(t)\|_{H^{s+2}}^{4}\right)\right] .
$$

The estimate of the last term, given by (2.18), simply follows from the boundedness of the operator $T_{n, \delta t}$ in $H^{s}\left(\mathbb{R}^{d}\right)$ and the fact that the function $g_{k}$ is Lipschitz in $H^{s}\left(\mathbb{R}^{d}\right) \times H^{s}\left(\mathbb{R}^{d}\right)$;

$$
\mathbb{E}\left(\left\|e_{n, \delta t, 5}\right\|_{H^{s}}^{2}\right) \leq C(\delta t)^{2} \mathbb{E}\left(\left\|e_{n}\right\|_{H^{s}}^{2}+\left\|e_{n+1}\right\|_{H^{s}}^{2}\right) .
$$

We now wish to estimate the second term in (2.19), i.e. to consider the $H^{s}$-inner products of the terms $e_{n, \delta t, j}$, for $1 \leq j \leq 5$. We first note that all the terms of the form $\mathbb{E}\left(e_{n, \delta t, j}, e_{n, \delta t, k}\right)_{s}$ with $2 \leq j, k \leq 4$ may be bounded thanks to the Cauchy-Schwarz inequality, since from $(2.21),(2.29)$ and $(2.32)$, for any $j$ with $2 \leq j \leq 4$,

$$
\mathbb{E}\left(\left\|e_{n, \delta t, j}\right\|_{H^{s}}^{2}\right) \leq C(\delta t)^{3}\left[1+\sup _{t \in[0, T]} \mathbb{E}\left(\|u(t)\|_{H^{s+6}}^{4}\right)\right] .
$$


Let us estimate the expectation of the product of the first and second terms in (2.19). Using the expressions of those terms given in (2.14) and (2.15), together with Plancherel equality and the Fourier transform of the operators $S_{n, \delta t}$ and $S\left(t_{n+1}, t_{n}\right)$, we obtain

$$
\begin{aligned}
\mathbb{E}\left(e_{n, \delta t, 1}, e_{n, \delta t, 2}\right)_{s} & =\mathbb{E}\left(S_{n, \delta t} e_{n},\left(S_{n, \delta t}-S\left(t_{n+1}, t_{n}\right)\right) u\left(t_{n}\right)\right)_{s} \\
& =\mathbb{E}\left(e_{n}, S_{n, \delta t}^{*}\left(S_{n, \delta t}-S\left(t_{n+1}, t_{n}\right)\right) u\left(t_{n}\right)\right)_{s} \\
& =\mathcal{R} e \mathbb{E} \int_{\mathbb{R}^{d}}\left(1+|\xi|^{2}\right)^{s} \hat{e}_{n}(\xi)\left(1-e^{-2 i \arctan \left(\frac{\sqrt{\delta t}}{2} \chi_{n}|\xi|^{2}\right)+i \sqrt{\delta t} \chi_{n}|\xi|^{2}}\right) \overline{\hat{u}}\left(t_{n}, \xi\right) d \xi
\end{aligned}
$$

and since $\chi_{n}$ is independent of $\hat{u}\left(t_{n}, \xi\right)$, the previous term is equal to

$$
\mathcal{R} e \int_{\mathbb{R}^{d}} \mathbb{E}\left(1-e^{i \sqrt{\delta t} \chi_{n}|\xi|^{2}-2 i \arctan \left(\frac{\sqrt{\delta t}}{2} \chi_{n}|\xi|^{2}\right)}\right) \mathbb{E}\left(\left(1+|\xi|^{2}\right)^{s} \hat{e}_{n}(\xi) \overline{\hat{u}}\left(t_{n}, \xi\right)\right) d \xi .
$$

Since

$$
\arctan x-x=-\frac{x^{3}}{3}+x^{5} \varepsilon(x)
$$

for some bounded function $\varepsilon(x)$ on $\mathbb{R}$, it follows

$$
\begin{aligned}
& 1-e^{i \sqrt{\delta t} \chi_{n}|\xi|^{2}-2 i \arctan \left(\frac{\sqrt{\delta t}}{2} \chi_{n}|\xi|^{2}\right)} \\
= & 1-e^{\frac{i}{12}(\delta t)^{3 / 2} \chi_{n}^{3}|\xi|^{6}+i(\delta t)^{5 / 2} \chi_{n}^{5}|\xi|^{10} \varepsilon\left(\frac{\sqrt{\delta t}}{2} \chi_{n}|\xi|^{2}\right)} \\
= & -\frac{i}{12}(\delta t)^{3 / 2} \chi_{n}^{3}|\xi|^{6}+(\delta t)^{5 / 2} \chi_{n}^{5}|\xi|^{10} \eta\left(\sqrt{\delta t} \chi_{n}|\xi|^{2}\right),
\end{aligned}
$$

where, again, $\eta$ is a bounded function on $\mathbb{R}$. Now, since $\mathbb{E}\left(\chi_{n}^{3}\right)=0$, this and CauchySchwarz inequality easily imply

$$
\begin{aligned}
\left|\mathbb{E}\left(e_{n, \delta t, 1}, e_{n, \delta t, 2}\right)_{s}\right| & \leq C(\delta t)^{5 / 2} \mathbb{E}\left(\left\|e_{n}\right\|_{H^{s}}^{2}\right)^{1 / 2} \mathbb{E}\left(\left\|u\left(t_{n}\right)\right\|_{H^{s+5}}^{2}\right)^{1 / 2} \\
& \leq C_{1}(\delta t) \mathbb{E}\left(\left\|e_{n}\right\|_{H^{s}}^{2}\right)+C_{2}(\delta t)^{4} \sup _{t \in[0, T]} \mathbb{E}\left(\|u(t)\|_{H^{s+5}}^{2}\right) .
\end{aligned}
$$

We now consider the estimate of the expectation of the $H^{s}$ inner product of the first and third terms in (2.19). Using the expressions given in (2.14) and (2.16), we easily obtain

$$
\begin{aligned}
& \mathbb{E}\left(e_{n, \delta t, 1}, e_{n, \delta t, 3}\right)_{s} \\
= & \mathbb{E}\left(e_{n}, i S_{n, \delta t}^{*} \int_{t_{n}}^{t_{n+1}} S\left(t_{n+1}, s\right)\left(g_{k}\left(u\left(t_{n}\right), u\left(t_{n+1}\right)\right)-f_{k}(u(s))\right) d s\right)_{s} \\
= & I+I I+I I I
\end{aligned}
$$

with

$$
\begin{gathered}
I=\mathbb{E}\left(e_{n}, i \int_{t_{n}}^{t_{n+1}}\left[f_{k}\left(u\left(t_{n}\right)\right)-f_{k}(u(s))\right] d s\right)_{s}, \\
I I=(\delta t) \mathbb{E}\left(e_{n}, i\left[g_{k}\left(u\left(t_{n}\right), u\left(t_{n+1}\right)\right)-f_{k}\left(u\left(t_{n}\right)\right)\right]\right)_{s}
\end{gathered}
$$


and

$$
I I I=\mathbb{E}\left(e_{n}, i \int_{t_{n}}^{t_{n+1}}\left(I-S_{n, \delta t}^{*} S\left(t_{n+1}, s\right)\right)\left[g_{k}\left(u\left(t_{n}\right), u\left(t_{n+1}\right)\right)-f_{k}(u(s))\right] d s\right)_{s} .
$$

The use of a Taylor expansion for $f_{k}$ - which is easily seen to belong to $C_{b}^{2}\left(H^{s}\left(\mathbb{R}^{d}\right)\right)$ allows to write

$$
\begin{aligned}
I= & \mathbb{E}\left(e_{n}, i \int_{t_{n}}^{t_{n+1}} D f_{k}\left(u\left(t_{n}\right)\right) \mathcal{E}_{n}(s) d s\right)_{s} \\
& +\mathbb{E}\left(e_{n}, i \int_{t_{n}}^{t_{n+1}} \int_{0}^{1}(1-\theta) D^{2} f_{k}\left(u\left(t_{n}\right)+\theta \mathcal{E}_{n}(s)\right)\left(\mathcal{E}_{n}(s), \mathcal{E}_{n}(s)\right) d \theta d s\right)_{s} \\
= & I_{1}+I_{2} .
\end{aligned}
$$

Thanks to the Cauchy-Schwarz inequality and the fact that

$$
\mathbb{E}\left(\left\|\mathcal{E}_{n}(s)\right\|_{H^{s}}^{4}\right) \leq C(\delta t)^{2} \sup _{t \in[0, T]} \mathbb{E}\left(\|u(t)\|_{H^{s+2}}^{4}\right),
$$

which follows easily from Lemma 2.8 and (2.25), the following estimate holds :

$$
I_{2} \leq C(\delta t)^{2} \mathbb{E}\left(\left\|e_{n}\right\|_{H^{s}}^{2}\right)^{1 / 2} \sup _{t \in[0, T]} \mathbb{E}\left(\|u(t)\|_{H^{s+2}}^{4}\right)^{1 / 2}
$$

In order to estimate the term $I_{1}$, we use again, the expression $(2.25)$ of $\mathcal{E}_{n}$ which allows to write

$$
\begin{aligned}
& \mathbb{E}\left(e_{n}, \int_{t_{n}}^{t_{n+1}} D f_{k}\left(u\left(t_{n}\right)\right) \mathcal{E}_{n}(s) d s\right)_{s} \\
= & \mathbb{E}\left(e_{n}, \int_{t_{n}}^{t_{n+1}} D f_{k}\left(u\left(t_{n}\right)\right)\left(S\left(s, t_{n}\right)-I\right) u\left(t_{n}\right) d s\right)_{s} \\
& +\mathbb{E}\left(e_{n}, \int_{t_{n}}^{t_{n+1}} D f_{k}\left(u\left(t_{n}\right)\right) \cdot i \int_{t_{n}}^{s} S(s, \sigma) f_{k}(u(\sigma)) d \sigma d s\right)_{s} .
\end{aligned}
$$

The second term in the right hand side above is bounded, again thanks to the CauchySchwarz inequality, by

$$
C(\delta t)^{2} \mathbb{E}\left(\left\|e_{n}\right\|_{H^{s}}^{2}\right)^{1 / 2} \sup _{t \in[0, T]} \mathbb{E}\left(\|u(t)\|_{H^{s}}^{2}\right)^{1 / 2}
$$

The first term is equal to

$$
\mathbb{E}\left(e_{n}, \int_{t_{n}}^{t_{n+1}} D f_{k}\left(u\left(t_{n}\right)\right) \mathbb{E}\left(S\left(s, t_{n}\right)-I\right) u\left(t_{n}\right) d s\right)_{s}
$$

and the use of Cauchy-Schwarz inequality, together with the fact that $D f_{k}(u)$ is a bounded operator in $H^{s}\left(\mathbb{R}^{d}\right)$ uniformly in $u$, allows to bound this term by

$$
C(\delta t)^{1 / 2} \mathbb{E}\left(\left\|e_{n}\right\|_{H^{s}}^{2}\right)^{1 / 2} \mathbb{E}\left(\int_{t_{n}}^{t_{n+1}}\left\|\mathbb{E}\left(S\left(s, t_{n}\right)-I\right) u\left(t_{n}\right)\right\|_{H^{s}}^{2} d s\right)^{1 / 2} .
$$


Then, Parseval Theorem and the fact that, or all $s \in\left(t_{n}, t_{n+1}\right)$,

$$
\begin{aligned}
\left|\mathbb{E}\left(\mathcal{F}_{x}\left(\left(S\left(s, t_{n}\right)-I\right) u\left(t_{n}\right)\right)(\xi) \mid \mathcal{F}_{t_{n}}\right)\right| & =\left|\mathbb{E}\left(e^{i\left(\beta(s)-\beta\left(t_{n}\right)\right)|\xi|^{2}}-1\right) \hat{u}\left(t_{n}, \xi\right)\right| \\
& =\left|e^{-\left(s-t_{n}\right)|\xi|^{4} / 2}-1\right|\left|\hat{u}\left(t_{n}, \xi\right)\right| \\
& \leq C(\delta t)|\xi|^{4}\left|\hat{u}\left(t_{n}, \xi\right)\right|
\end{aligned}
$$

lead to the estimate :

$$
I_{1} \leq C(\delta t)^{2} \mathbb{E}\left(\left\|e_{n}\right\|_{H^{s}}^{2}\right)^{1 / 2} \sup _{t \in[0, T]} \mathbb{E}\left(\|u(t)\|_{H^{s+4}}^{2}\right)^{1 / 2}
$$

The term $I I$ in (2.36) may be estimated in the same way. In order to bound the third term, we use the following lemma.

Lemma 2.9. There exists a constant $C>0$ such that for any random function $v \in$ $L^{4}\left(\Omega ; H^{s+2}\left(\mathbb{R}^{d}\right)\right)$, and for any $s \in\left(t_{n}, t_{n+1}\right)$,

$$
\mathbb{E}\left(\left\|\left(I-S_{n, \delta t}^{*} S\left(t_{n+1}, s\right)\right) v\right\|_{H^{s}}^{2}\right) \leq C(\delta t)\left(\mathbb{E}\left(\|v\|_{H^{s+2}}^{4}\right)\right)^{1 / 2} .
$$

Applying Lemma 2.9 with $v=g_{k}\left(u\left(t_{n}\right), u\left(t_{n+1}\right)\right)-f_{k}(u(s))$ and using $(2.23)$, together with Cauchy-Schwarz inequality and (2.27)-(2.28) leads yo

$$
I I I \leq C(\delta t)^{2} \mathbb{E}\left(\left\|e_{n}\right\|_{H^{s}}^{2}\right)^{1 / 2} \sup _{t \in[0, T]} \mathbb{E}\left(\|u(t)\|_{H^{s+4}}^{4}\right)^{1 / 2} .
$$

We deduce from $(2.42),(2.44)$, the same estimate for $I I$ and $(2.45)$, that

$$
\mathbb{E}\left(e_{n, \delta t, 1}, e_{n, \delta t, 3}\right)_{s} \leq C(\delta t)^{2} \mathbb{E}\left(\left\|e_{n}\right\|_{H^{s}}^{2}\right)^{1 / 2}\left[1+\sup _{t \in[0, T]} \mathbb{E}\left(\|u(t)\|_{H^{s+4}}^{4}\right)\right]
$$

Proof of Lemma 2.9. The proof is based on similar arguments as those of Lemma 2.7 : Parseval theorem and the fact that $\left|1-e^{i x}\right|^{2} \leq C|x|^{2}$ for any real $x$ allows to write, for any $v \in L^{4}\left(\Omega ; H^{s+2}\left(\mathbb{R}^{d}\right)\right)$,

$$
\begin{aligned}
& \mathbb{E}\left(\left\|\left(I-S_{n, \delta t}^{*} S\left(t_{n+1}, s\right)\right) v\right\|_{H^{s}}^{2}\right) \\
& \leq\left.\left. C \mathbb{E} \int_{\mathbb{R}^{d}}\left|2 \arctan \left(\frac{\sqrt{\delta t}}{2} \chi_{n}|\xi|^{2}\right)+\left(\beta\left(t_{n+1}\right)-\beta(s)\right)\right| \xi\right|^{2}\right|^{2}\left(1+|\xi|^{2}\right)^{s}|\hat{v}(\xi)|^{2} d \xi \\
& \leq C(\delta t)\left(\mathbb{E}\left(\chi_{n}^{2}+\frac{\left|\beta\left(t_{n+1}\right)-\beta(s)\right|^{2}}{t_{n+1}-s}\right)^{2}\right)^{1 / 2} \mathbb{E}\left(\|v\|_{H^{s+2}}^{4}\right)^{1 / 2}
\end{aligned}
$$

by Cauchy-Schwarz inequality.

In order to estimate the term $\mathbb{E}\left(e_{n, \delta t, 1}, e_{n, \delta t, 4}\right)_{s}$, we use $(2.14),(2.17)$ and a Taylor expansion to decompose it as

$$
\mathbb{E}\left(e_{n, \delta t, 1}, e_{n, \delta t, 4}\right)_{s}=I+I I+I I I
$$

with

$$
I=\mathbb{E}\left(e_{n}, i S_{n, \delta t}^{*}\left[(\delta t) T_{n, \delta t}-\int_{t_{n}}^{t_{n+1}} S\left(t_{n+1}, s\right) d s\right] f_{k}\left(u\left(t_{n}\right)\right)\right)_{s}
$$




$$
I I=\mathbb{E}\left(e_{n}, i S_{n, \delta t}^{*}\left[(\delta t) T_{n, \delta t}-\int_{t_{n}}^{t_{n+1}} S\left(t_{n+1}, s\right) d s\right] D_{v} g_{k}\left(u\left(t_{n}\right), u\left(t_{n}\right)\right) \mathcal{E}_{n}\left(t_{n+1}\right)\right)_{s}
$$

and

$$
I I I=\mathbb{E}\left(e_{n}, i S_{n, \delta t}^{*}\left[(\delta t) T_{n, \delta t}-\int_{t_{n}}^{t_{n+1}} S\left(t_{n+1}, s\right) d s\right] \int_{0}^{1}(1-\theta) D_{v v}^{2} g_{k}\left(u\left(t_{n}\right), u\left(t_{n}\right)\right)\left(\mathcal{E}_{n}\left(t_{n+1}\right)\right)^{2} d \theta\right)_{s}
$$

where $\mathcal{E}_{n}$ is defined in (2.25). The two last terms are bounded as above, using CauchySchwarz inequality and the fact that $(\delta t) T_{n, \delta t}-\int_{t_{n}}^{t_{n+1}} S\left(t_{n+1}, s\right) d s$ is of order $(\delta t)^{3 / 2}$ while $\mathcal{E}_{n}\left(t_{n+1}\right)$ is of order $(\delta t)^{1 / 2}$ (see $(2.27)$ and $\left.(2.41)\right)$. The first term is bounded thanks to arguments similar to those we have used to obtain (2.35). Indeed, Plancherel Theorem implies

$$
I=\mathbb{E} \mathcal{I} m \int_{\mathbb{R}^{d}}\left(1+|\xi|^{2}\right)^{s} \overline{\hat{e}}_{n}(\xi) \mathcal{F}_{x}\left[\mathbb{E}\left(S_{n, \delta t}^{*}\left((\delta t) T_{n, \delta t}-\int_{t_{n}}^{t_{n+1}} S\left(t_{n+1}, s\right) d s\right) f_{k}\left(u\left(t_{n}\right)\right)\right)\right] d \xi .
$$

Now, for a fixed $\xi \in \mathbb{R}^{d}$,

$$
\begin{aligned}
& \mathcal{F}_{x}\left[\mathbb{E}\left(S_{n, \delta t}^{*}\left((\delta t) T_{n, \delta t}-\int_{t_{n}}^{t_{n+1}} S\left(t_{n+1}, s\right) d s\right)\right) f_{k}\left(u\left(t_{n}\right)\right)\right](\xi) \\
= & \left(m_{1, n, \delta t}(\xi)+m_{2, n, \delta t}(\xi)\right) f_{k}\left(u\left(t_{n}\right)\right)(\xi)
\end{aligned}
$$

where

$$
m_{1, n, \delta t}(\xi)=(\delta t) \mathbb{E}\left[\left(1-i \frac{\sqrt{\delta t}}{2} \chi_{n}|\xi|^{2}\right)^{-1}-1\right]
$$

and

$$
m_{2, n, \delta t}(\xi)=\int_{t_{n}}^{t_{n+1}} \mathbb{E}\left(1-e^{-2 i \arctan \left(\frac{\sqrt{\delta t}}{2} \chi_{n}|\xi|^{2}\right)+i\left(\beta\left(t_{n+1}\right)-\beta(s)\right)|\xi|^{2}}\right) d s .
$$

On the one hand, we use the fact that

$$
\frac{1}{1-i x}-1=i x+x^{2} \varepsilon(x)
$$

for some bounded function $\varepsilon$ on $\mathbb{R}$ to obtain

$$
\left|m_{1, n, \delta t}(\xi)\right| \leq C(\delta t)^{2}|\xi|^{4} .
$$

On the other hand, we successively use the equalities

$$
\arctan x=x+x^{3} \eta(x)
$$

and

$$
1-e^{i y}=-i y+y^{2} \nu(y)
$$

for some bounded functions $\eta$ and $\nu$ on $\mathbb{R}$, to get

$$
\left|m_{2, n, \delta t}(\xi)\right| \leq C(\delta t)^{2}|\xi|^{4} .
$$

We deduce from $(2.47),(2.48),(2.49)$ and Cauchy-Schwarz inequality that

$$
I \leq C(\delta t)^{2} \mathbb{E}\left(\left\|e_{n}\right\|_{H^{s}}^{2}\right)^{1 / 2} \mathbb{E}\left(\left\|f_{k}\left(u\left(t_{n}\right)\right)\right\|_{H^{s+4}}^{2}\right)^{1 / 2} .
$$


Because $s$ is a positive integer, one may easily check that there is a constant $C(k)$ such that

$$
\left\|f_{k}(v)\right\|_{H^{s+4}} \leq C(k)\|v\|_{H^{s+4}}^{2}, \quad \forall v \in H^{s+4}\left(\mathbb{R}^{d}\right)
$$

and

$$
I \leq C(\delta t)^{2} \mathbb{E}\left(\left\|e_{n}\right\|_{H^{s}}^{2}\right)^{1 / 2} \sup _{t \in[0, T]}\left(\mathbb{E}\left(\|u(t)\|_{H^{s+4}}^{2}\right)\right)^{1 / 2}
$$

It follows

$$
\mathbb{E}\left(e_{n, \delta t, 1}, e_{n, \delta t, 4}\right)_{s} \leq C(\delta t)^{2} \mathbb{E}\left(\left\|e_{n}\right\|_{H^{s}}^{2}\right)^{1 / 2}\left[\sup _{t \in[0, T]} \mathbb{E}\left(\|u(t)\|_{H^{s+4}}^{4}\right)+1\right]
$$

The inner product of the first and fifth terms in (2.19) is easily estimated thanks to the Cauchy-Schwarz inequality, the fact that $g_{k}$ is a Lipschitz function in $H^{s} \times H^{s}$, and the unitarity of the operators $S_{n, \delta t}^{*}$ and $T_{n, \delta t}$ in $H^{s}\left(\mathbb{R}^{d}\right)$ :

$$
\begin{aligned}
& \mathbb{E}\left(e_{n, \delta t, 1}, e_{n, \delta t, 5}\right)_{s} \\
= & (\delta t) \mathbb{E}\left(e_{n}, i S_{n, \delta t}^{*} T_{n, \delta t}\left[g\left(u_{n}, u_{n+1}\right)-g\left(u\left(t_{n}\right), u\left(t_{n+1}\right)\right)\right]\right)_{s} \\
\leq & C(\delta t) \mathbb{E}\left(\left\|e_{n}\right\|_{H^{s}}^{2}\right)^{1 / 2} \mathbb{E}\left(\left\|e_{n}\right\|_{H^{s}}^{2}+\left\|e_{n+1}\right\|_{H^{s}}^{2}\right)^{1 / 2} \\
\leq & C(\delta t) \mathbb{E}\left(\left\|e_{n}\right\|_{H^{s}}^{2}+\left\|e_{n+1}\right\|_{H^{s}}^{2}\right) .
\end{aligned}
$$

Finally, all terms of the form $\mathbb{E}\left(e_{n, \delta t, j}, e_{n, \delta t, 5}\right)$ with $2 \leq j \leq 5$ are bounded using Cauchy Schwarz inequality and (2.21), (2.29), (2.32) and (2.33). It follows

$$
\mathbb{E}\left(e_{n \delta t, j}, e_{n, \delta t, 5}\right)_{s} \leq C(\delta t)^{5 / 2} \mathbb{E}\left(\left\|e_{n}\right\|_{H^{s}}^{2}+\left\|e_{n+1}\right\|_{H^{s}}^{2}\right)^{1 / 2} \mathbb{E}\left(\left\|e_{n}\right\|_{H^{s}}^{2}\right)^{1 / 2}
$$

Gathering then (2.19)-(2.21), (2.29), (2.32)-(2.35), (2.46), (2.50), (2.51) and (2.52), we deduce the existence of constants $\tilde{K}_{j}$, for $j=1,2,3$, depending on $k$ and $\sup _{t \in[0, T]} \mathbb{E}\left(\|u(t)\|_{H^{s+6}}^{4}\right)$ (which also depends on $k$ ) such that for any $n$ with $n \delta t \leq T$,

$$
\mathbb{E}\left(\left\|e_{n+1}\right\|_{H^{s}}^{2}\right) \leq\left(1+\tilde{K}_{1} \delta t\right) \mathbb{E}\left(\left\|e_{n}\right\|_{H^{s}}^{2}\right)+\tilde{K}_{2}(\delta t)^{3}+\tilde{K}_{3}(\delta t) \mathbb{E}\left(\left\|e_{n+1}\right\|_{H^{s}}^{2}\right) .
$$

We deduce that (2.11) holds provided $T / N=\delta t \leq 1 / 2 \tilde{K}_{3}$ with $K_{1}=3 \tilde{K}_{1}+2 \tilde{K}_{3}$ and $K_{2}=2 \tilde{K}_{2}$. The discrete Gronwall Lemma concludes the proof of Proposition 2.6 for $\delta t=T / N \leq 1 / 2 \tilde{K}_{3}$. The conclusion is obviously still true for all $N<2 T \tilde{K}_{3}$, since the continuous solution $u$ has paths a.s. in $C\left([0, T] ; H^{s}\left(\mathbb{R}^{d}\right)\right)$ and the discrete solution $u_{\delta t}$ given by Proposition 2.2 has paths a.s. in $L^{\infty}\left(0, T ; H^{s}\left(\mathbb{R}^{d}\right)\right)$.

2.3. Proof of Theorem 2.3. We recall that the discrete solution is now defined by $u_{n}^{\delta t}=u_{n}^{k}$ if $n \delta t \leq \tau_{\delta t}^{k}$, and $\tau_{\delta t}^{k}$ is defined in (2.8), for any $k \in \mathbb{N}$. On the other hand, by Proposition (2.6), we have for any $k \in \mathbb{N}$, recalling that $\delta t=T / N$ :

$$
\mathbb{E}\left(\sup _{n \leq N}\left\|u^{k}\left(t_{n}\right)-u_{n}^{k}\right\|_{H^{s}}^{2}\right) \leq \sum_{n \leq N} \mathbb{E}\left(\left\|u^{k}\left(t_{n}\right)-u_{n}^{k}\right\|_{H^{s}}^{2}\right) \leq C(k) T(\delta t)
$$


and this show that $\sup _{n \leq N}\left\|u\left(t_{n}\right)-u_{n}^{\delta t}\right\|_{H^{s}}$ converges to zero in probability as $\delta t$ tends to zero : indeed, if $\varepsilon>0$ is fixed, with $\varepsilon \leq 1$, then for $k \in \mathbb{N}$,

$$
\begin{aligned}
& \mathbb{P}\left(\sup _{n \leq N}\left\|u\left(t_{n}\right)-u_{n}^{\delta t}\right\|_{H^{s}} \geq \varepsilon\right) \\
\leq & \mathbb{P}\left(\sup _{n \leq N}\left\|u\left(t_{n}\right)\right\|_{H^{s}} \geq k\right)+\mathbb{P}\left(\sup _{n \leq N}\left\|u^{k+1}\left(t_{n}\right)-u_{n}^{k+1}\right\|_{H^{s}} \geq \varepsilon\right) \\
\leq & \mathbb{P}\left(\sup _{n \leq N}\left\|u\left(t_{n}\right)\right\|_{H^{s}} \geq k\right)+T C(k)(\delta t) \varepsilon^{-2} .
\end{aligned}
$$

Hence, choosing first $k$ sufficiently large so that $\mathbb{P}\left(\sup _{n \leq N}\left\|u\left(t_{n}\right)\right\|_{H^{s}} \geq k\right) \leq \varepsilon / 2-$ this is possible since $u \in C\left([0, T] ; H^{s}\left(\mathbb{R}^{d}\right)\right)$ a.s. - then $\delta t$ sufficiently small so that $T C(k)(\delta t) \varepsilon^{-2} \leq \varepsilon / 2$, the convergence in probability is proved.

We deduce from this convergence in probability, taking again $\delta t=T / N$ for some $N \in \mathbb{N}$, that

$$
\lim _{K \rightarrow \infty} \mathbb{P}\left(\sup _{n \leq N}\left\|u_{n}^{\delta t}\right\|_{H^{s}} \geq K\right)=0
$$

uniformly with respect to $\delta t=T / N$. Now, we can prove that $\mathbb{P}\left(\left\|u\left(t_{n}\right)-u_{n}^{\delta t}\right\|_{H^{s}} \geq C \delta t\right)$ tends to zero as $C$ tends to infinity, uniformly with respect to $\delta t$ : indeed, thanks to Markov inequality

$$
\begin{aligned}
& \mathbb{P}\left(\left\|u\left(t_{n}\right)-u_{n}^{\delta t}\right\|_{H^{s}} \geq C \delta t\right) \\
\leq & \mathbb{P}\left(\sup _{n \leq N}\left\|u\left(t_{n}\right)\right\|_{H^{s}} \geq k\right)+\mathbb{P}\left(\sup _{n \leq N}\left\|u_{n}^{\delta t}\right\|_{H^{s}} \geq k\right)+\frac{\mathbb{E}\left(\left\|u^{k}\left(t_{n}\right)-u_{n}^{k}\right\|_{H^{s}}^{2}\right)}{C^{2}(\delta t)^{2}} .
\end{aligned}
$$

We conclude choosing first $k$ sufficiently large so that the two first terms are small, thanks to (2.53), then for $k$ fixed, choosing $C$ sufficiently large, so that the last term, which is bounded by $C(k) / C^{2}$ thanks to Proposition 2.6, is in turn small.

\section{Numerical simulations in Dimension OnE}

In this section, we consider equation (1.1) in space dimension one, i.e.

$$
i d \phi+\partial_{x}^{2} \phi \circ d \beta+|\phi|^{2 \sigma} \phi=0
$$

for different values of $\sigma$. We numerically illustrate the order of convergence given in Section 2, and we investigate the behavior of the solutions in terms of the power $\sigma$ of the nonlinear term.

3.1. Numerical schemes. Three different schemes have been used for the numerical simulations. These are classical schemes for the deterministic nonlinear Schrödinger equation, that we have adapted to the simulation of the equation with Stratonovich noise (3.1). For each scheme, we work on the spatial domain $\left[-L_{x}, L_{x}\right]$ and we use a regular mesh in space. We of course need the use of boundary conditions in space, even though our aim is to approximate the solution of equation (3.1) set in $\mathbb{R}$. We will work with either Dirichlet or periodic boundary conditions, and take $L_{x}$ sufficient large so 
that the solution stays confined in the interval $\left[-L_{x}, L_{x}\right]$ up to the final time of the computations. The spatial mesh step size is $\delta x=\frac{2 L_{x}}{N_{x}}$ where $N_{x}=2 M_{x}$ is an even integer, the time step being $\delta t>0$, and we denote the grid points in space and time by

$$
x_{j}=-L_{x}+j \delta x, \quad j=0,1, \ldots, N_{x}, \quad \text { and } t_{k}=k \delta t, \quad k=0,1,2 \ldots
$$

with $x_{0}=-L_{x}, x_{N_{x}}=L_{x}$. Furthermore, $\phi_{i}^{k}$ is the approximations of $\phi\left(t_{k}, x_{i}\right)$.

Time-splitting spectral discretization. We consider the equation with periodic boundary conditions, and use pseudo-spectral discretization in space. The relations between $\phi_{i}^{k}$ and its discrete Fourier coefficients in space $\hat{\phi}_{\ell}^{k}$ are given by

$$
\phi_{i}^{k}=\sum_{\ell=-M_{x}}^{M_{x}-1} \hat{\phi}_{\ell}^{k} e^{i \xi_{\ell} x_{i}}, \text { for } i=1, \cdots N_{x}
$$

and

$$
\widehat{\phi}_{\ell}^{k}=\frac{1}{N_{x}} \sum_{i=1}^{N_{x}} \phi_{i}^{k} e^{-i \xi_{\ell} x_{i}}, \quad \ell=-M_{x}, . ., M_{x}-1
$$

where $\xi_{\ell}=\frac{\pi \ell}{L_{x}}$, for $\ell=-M_{x}, \ldots, 0, \ldots, M_{x}-1$, gives the regular mesh grid for the frequencies.

Equation (3.1) is solved with the classical Strang splitting scheme. One first solves

$$
i d \phi+\partial_{x}^{2} \phi \circ d \beta=0,
$$

on a time interval of length $\delta t / 2$. In the second step, one solves

$$
\left\{\begin{array}{l}
i \partial_{t} \phi+|\phi|^{2 \sigma} \phi=0 \\
\phi_{0}=\phi^{*}
\end{array}\right.
$$

where $\phi^{*}$ is the solution of (3.4) at time $t=t_{k}+\delta t / 2$. The last step consists in solving again (3.4) over a half time step with initial data given by the solution of the second step.

Equation (3.4) is discretized in space by the Fourier spectral method and integrated exactly in time. For $t \in\left[t_{k}, t_{k+1}\right]$, multiplying (3.5) by $\bar{\phi}$, the conjugate of $\phi$ and taking the imaginary part, we obtain for $x \in\left[-L_{x}, L_{x}\right]$ and $t \geq 0$ :

$$
\frac{d}{d t}|\phi(t, x)|^{2}=0
$$

With this conservation law, solving (3.5), amounts to solve :

$$
i \partial_{t} \phi+\left|\phi^{*}\right|^{2 \sigma} \phi=0
$$

Hence the algorithm may be summarized as follows. 
For the first step of the splitting, we compute

$$
\begin{aligned}
\widehat{\phi}_{l}^{k} & =\frac{1}{N_{x}} \sum_{i=1}^{N_{x}} \phi_{i}^{k} e^{-i \xi_{l} x_{i}}, \quad l=-M_{x}, . ., M_{x}-1, \\
\widehat{\phi}_{l}^{*} & =\widehat{\phi}_{l}^{k} \exp \left(-i \sqrt{\frac{\delta t}{2}} \xi_{l}^{2} \chi_{k}\right) \\
\phi_{i}^{*} & =\sum_{l=-M_{x}}^{M_{x}-1} \hat{\phi}_{l}^{*} e^{i \xi_{l} x_{i}}, \text { for } i=1, \cdots N_{x},
\end{aligned}
$$

where $\sqrt{\frac{\delta t}{2}} \chi_{k}=\beta\left(t_{k}+\frac{\delta t}{2}\right)-\beta\left(t_{k}\right)$, so that $\left(\chi_{k}\right)_{k=0,1, \ldots}$ is a family of independent normal random variables, and $\left(\widehat{U}_{l}\right)$ denote the Fourier coefficients of a periodic vector $U=\left(U_{i}\right)$.

For the second step, we compute

$$
\widetilde{\phi}_{j}^{k+1}=\phi_{j}^{*} \exp \left(i \delta t\left|\phi^{*}\right|^{2 \sigma}\right) .
$$

Finally for the last step :

$$
\left.\phi_{i}^{k+1}=\sum_{l=-M_{x}}^{M_{x}-1} \widehat{\widetilde{\phi}}_{l}^{k+1} \exp \left(i\left(\xi_{l} x_{i}-\sqrt{\frac{\delta t}{2}} \xi_{l}^{2} \chi_{k}\right)\right)\right) \text {, for } i=1, \cdots N_{x} .
$$

Crank-Nicolson scheme. We consider the Crank-Nicolson scheme which was analyzed in Section 2, together with finite differences centered discretization in space. We use here Dirichlet boundary conditions which are easily discretized with finite differences. Therefore, $\partial_{x}^{2}$ is approximated by the classical centered finite difference operator $D_{+} D_{-}$:

$$
\left(D_{+} D_{-} \phi\right)_{i}=\frac{\phi_{i+1}-2 \phi_{i}+\phi_{i-1}}{(\delta x)^{2}}
$$

The scheme reads:

$$
i \frac{\phi^{k+1}-\phi^{k}}{\delta t}+\frac{\chi_{k}}{\sqrt{\delta t}} D_{+} D_{-}\left(\frac{\phi^{k+1}+\phi^{k}}{2}\right)+g\left(\phi^{k+1}, \phi^{k}\right)=0
$$

where here $\sqrt{\delta t} \chi_{k}=\beta\left(t_{k}+\delta t\right)-\beta\left(t_{k}\right)$, and $g$ is defined by (2.4). Note that the computation of $\phi^{k+1}$ requires the resolution of a nonlinear problem for which a fixed point procedure is used.

Crank-Nicolson scheme with relaxation. For the last scheme, we use a fractional-step Crank-Nicolson scheme with relaxation introduced by C. Besse for the deterministic nonlinear Schrödinger equation [4]. The advantage is that the scheme is linearly implicit and requires only the resolution of a linear system. It reads in our case :

$$
i \frac{\phi^{k+1}-\phi^{k}}{\delta t}+\frac{\chi_{k}}{\sqrt{\delta t}} D_{+} D_{-}\left(\frac{\phi^{k+1}+\phi^{k}}{2}\right)+R^{k+\frac{1}{2}}\left(\frac{\phi^{k+1}+\phi^{k}}{2}\right)=0
$$


where again $\sqrt{\delta t} \chi_{k}=\beta\left(t_{k}+\delta t\right)-\beta\left(t_{k}\right)$, and the auxiliary function $R$ is given by

$$
\frac{R^{k+\frac{1}{2}}+R^{k-\frac{1}{2}}}{2}=\left|\phi^{k}\right|^{2 \sigma} .
$$

3.2. Numerical results. We here report the results of several numerical experiments. In order to avoid numerical reflections due to the boundaries, we take a domain $\left[-L_{x}, L_{x}\right]$ large enough.

In order to satisfy the required independence properties of the family of Gaussian random variables $\chi_{k}$, these are simulated with the Netlib Library (http://netlib.org) which uses a random generator routine with period $10^{32}-1$. This has to be compared with the maximum size of the random vector we use which is of order $10^{9}$.

3.2.1. Strong order of convergence. In the first experiment, we compute the strong numerical order of the three numerical schemes described above and in particular, we recover numerically the result obtained in Theorem 2.3. In the deterministic case, the schemes are known to be consistent and of order 2 in time (see [4], [5], [12] ) which is shown in figure 1.

We choose the computational domain $\left[-L_{x}, L_{x}\right]=[-30,30]$ and an initial data with a Gaussian profile $\phi(0, x)=\exp \left(-3 x^{2}\right)$. As our aim is to identify only the numerical order in time, we take a fine spatial mesh with $\delta x=10^{-3}$.

Since there is no explicit solution for the stochastic equation (3.1), we first compute a reference solution $\phi_{\text {ref }}$ on a fine mesh in time $\delta t_{\text {ref }}=10^{-5}$, that we compare to approximations of the same equation on 6 coarser grids in time by using a time step $\delta t=2^{p} \delta t_{\text {ref }}$ with $p=1, \ldots, 6$. The Brownian path (one trajectory) is kept fixed for each approximation. In figure 1, we plot four convergence curves corresponding to the logarithm of the relative errors $\sqrt{\left|\psi_{\text {ref }}-\psi_{2^{p} \delta t}\right|_{L^{2}}^{2}}$ at time $T=0.5$ for different schemes, in terms of the logarithm of the number of points (in time) used in the discretization. The slopes of the three curves corresponding to the stochastic schemes are close to -1 and we recover that the strong order of the Crank Nicolson scheme is 1 in time. Thus, the result agrees with the theoretical analysis of the previous section.

The same experiment has been done with many other trajectories and the same order of convergence has always been observed.

3.2.2. Influence of the power nonlinearities $\sigma$. We now investigate the influence of the power nonlinearity $\sigma$ on the behavior of the numerical solution of (3.1). For these experiments, we only use the Crank-Nicolson scheme for the following values of $\sigma: \sigma=1$, $\sigma=2, \sigma=3, \sigma=3.9$ and $\sigma=4$, which we conjecture to be the critical case. Indeed, due to the scaling invariance $\lambda^{-1 / 2} \beta(\lambda t)$ of the Brownian motion $\beta$, one may easily see that equation (3.1) with $\sigma=4$ is invariant under the scale change $u_{\lambda}(t, x)=\sqrt{\lambda} u\left(\lambda^{4} t, \lambda x\right)$ for any $\lambda>0$, ans so is the $L^{2}$-norm in dimension one. This suggests that equation (3.1) with $\sigma=4$ should be critical in $L^{2}(\mathbb{R})$. This is different from the deterministic case for which the $L^{2}$-critical power is $\sigma=2$. 


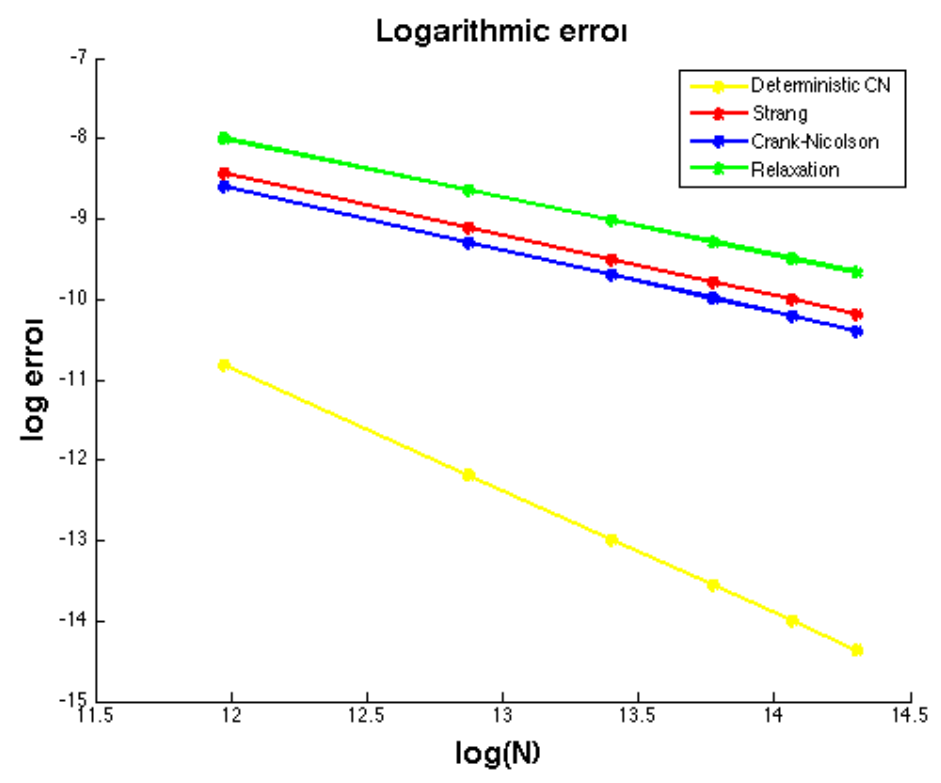

FiguRE 1. Strong order of convergence

For each simulation $(\sigma=1, \sigma=2, \sigma=3$ and $\sigma=3.9)$, we take a Gaussian initial state given by $\phi(0, x)=\exp \left(-3 x^{2}\right)$ on the computational domain $\left[-L_{x}, L_{x}\right]=[-10 \pi, 10 \pi]$ with $N_{x}=2^{12}$ points in space and a time step $\delta t=0.4 \delta x^{2}$ with a final time $T$ which is chosen in such a way that the numerical solution stays confined in $\left[-L_{x}, L_{x}\right]$ on the whole interval $[0, T]$. In order to understand the noise effects, all the experiments are done with one realization of a Brownian path. Note that all the different simulations that we have performed have given the same qualitative behavior.

Figure 2 shows the profile of the space-time evolution of the solution in the case $\sigma=1$. We can see clearly the effect of the noise on the dispersion. It seems that dispersion and nonlinearity stay well balanced for a while, resulting in alternating contractions and expansions of the solution. Here the solutions stays confined until $T=5$, then boundary effects occur (which would suggest that dispersion finally wins) and we had to stop the computation. The modulus of the Fourier modes of the solution are plot in figure 6 . In the cases $\sigma=2, \sigma=3$ and $\sigma=3.9$ (see figure 3, figure 4 and figure 5), the behavior of the solution in time is similar, although the final computation time may be different from one case to the other. The final amplitude seems much smaller than the initial amplitude although the modulus of the Fourier modes of the solution do not seem to move with time (see figure 6 and figure 7 ), which indicates that after a while there is no energy transfer between the different Fourier modes.

For the case $\sigma=4$, which is the expected critical case (see the above comments), it is much more difficult to perform numerical simulations since the numerical method has to be consistent with the small spatial scales of the blow up structures. Here we do not 

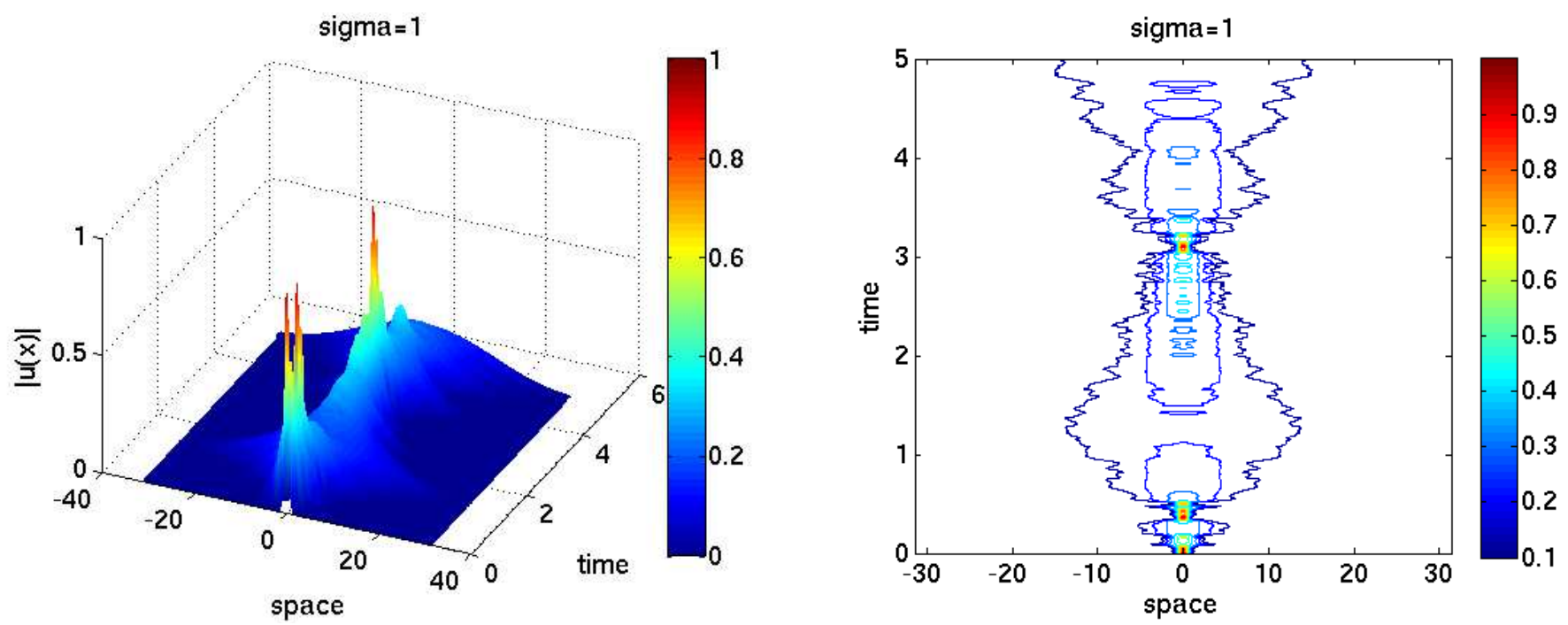

Figure 2. $\sigma=1$ : space-time evolution of the solution (left) and contour plot of the solution (right).
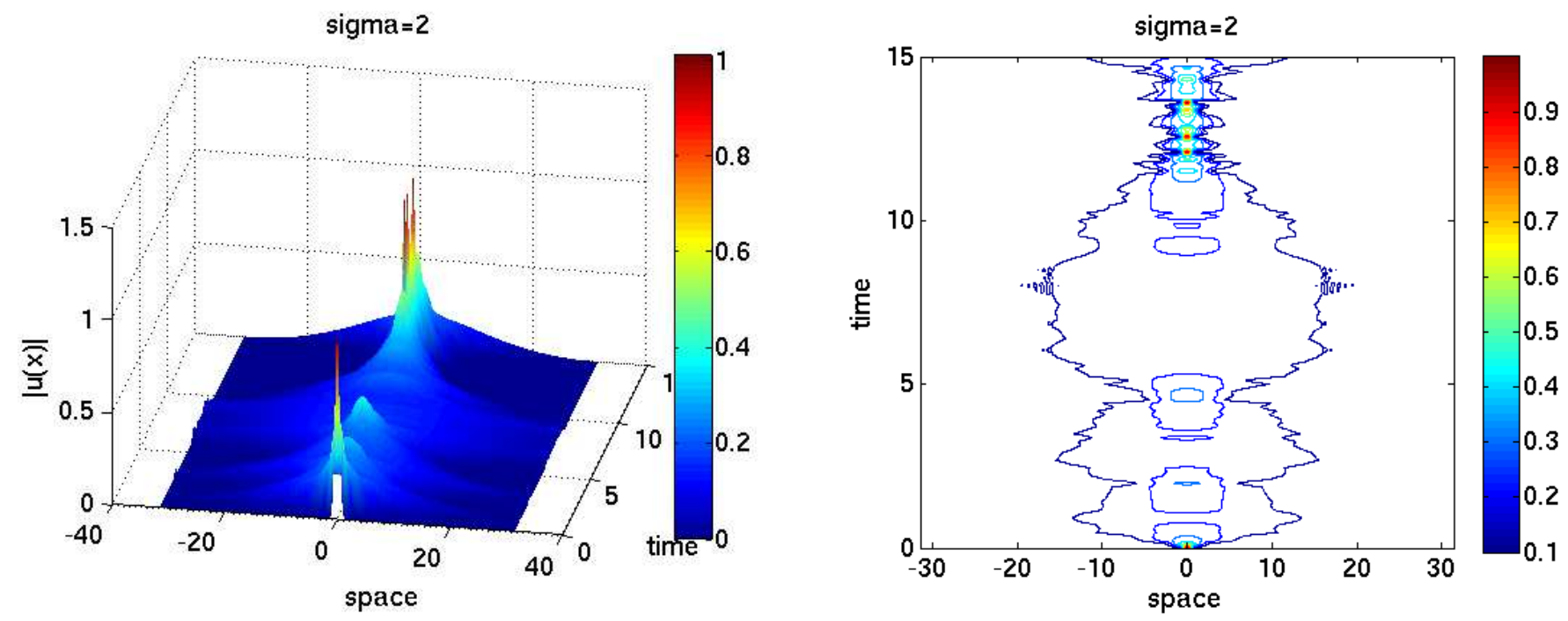

Figure 3. $\sigma=2$ : space-time evolution of the solution (left) and contour plot of the solution (right).

use spatial local refinement as in [10]. Since we are in dimension 1 in space, and it is not too expensive, we only refine globally the time-space discretization. We take here $\delta x=10^{-4}$ and $\delta t=0.1 \delta x^{2}$. We take a Gaussian inital data $\phi(0, x)=1.4 \exp \left(-3 x^{2}\right)$. In figure 8 we plot the modulus of the initial state and of the solution at time $t=0.035$. Figure 9 shows the modulus of the spatial spectrum of the solution at three different 

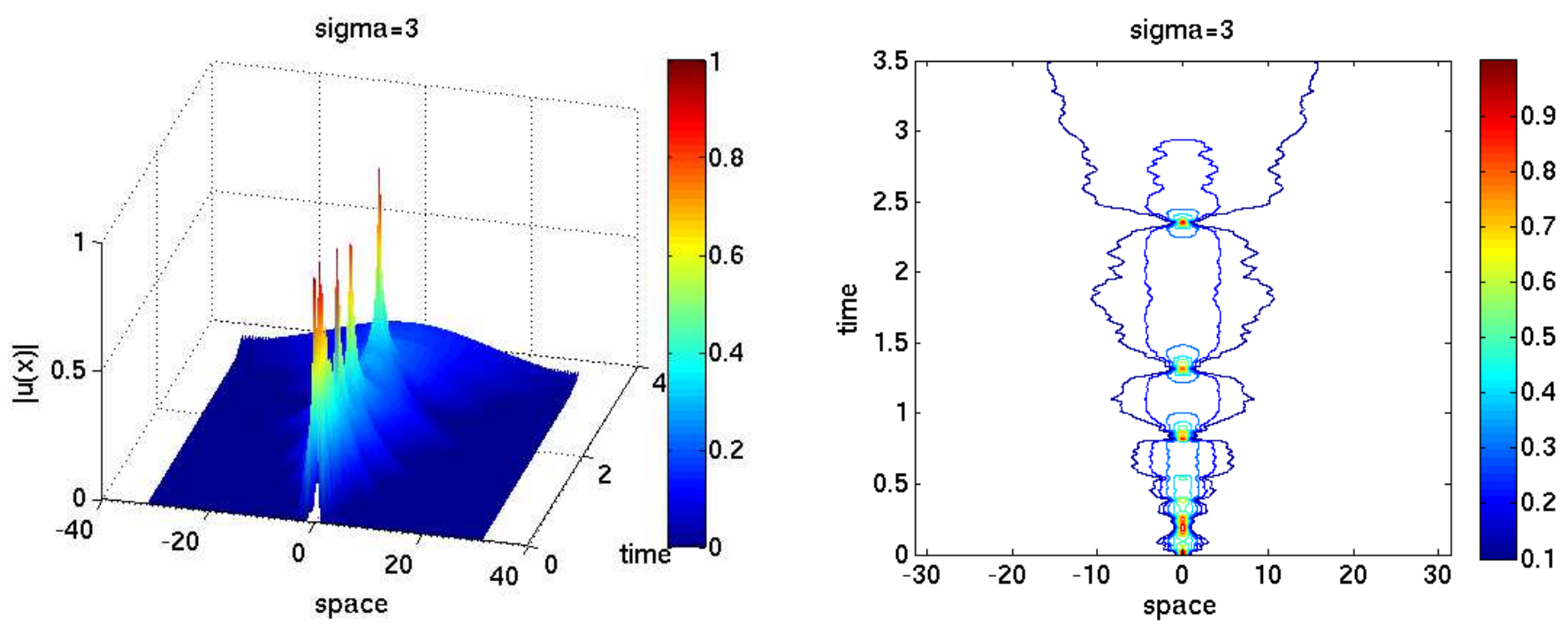

FigURE 4. $\sigma=3$ : space-time evolution of the solution (left) and contour plot of the solution (right).
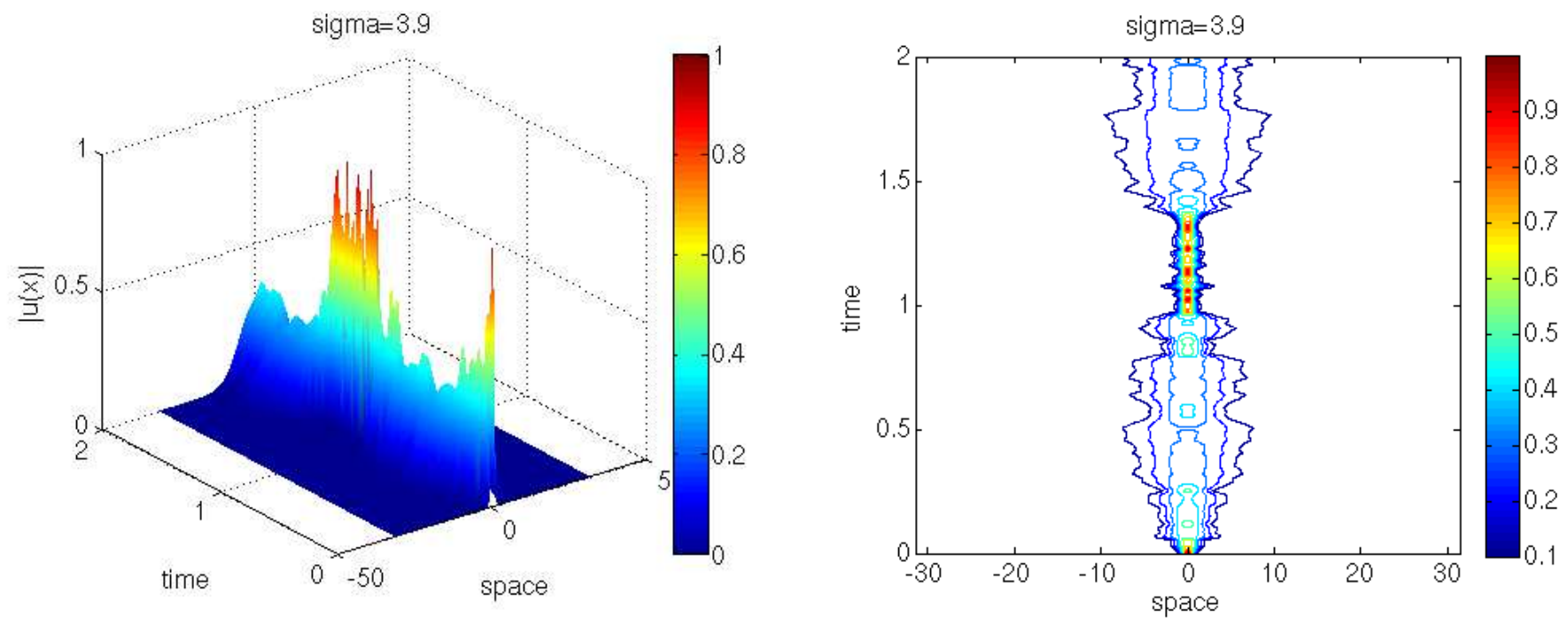

Figure 5. $\sigma=3.9$ : space-time evolution of the solution (left) and the contour plot of the solution (right).

times; there seems to be a collapse of this spatial spectrum at time $t=0.035$. Finally, figure 10 represents the evolution with time of the discrete $H^{1}$-norm, which indicates that the solution reaches very high amplitudes.

We finally plot in figure 11 the evolution with time of the expected amplitude of the solution for $\sigma=1$. We use the notation $<>$ for the empirical average which 


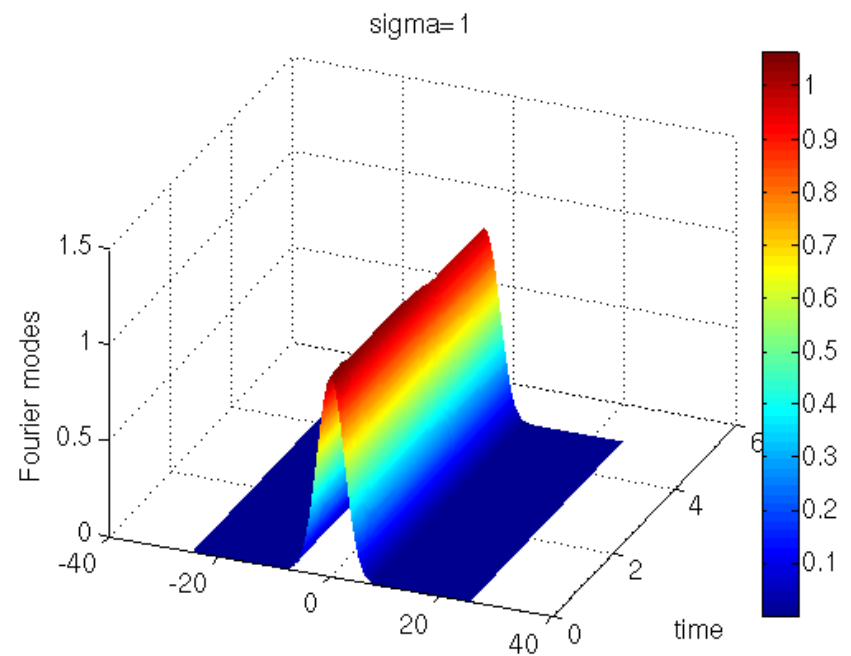

Fourier space

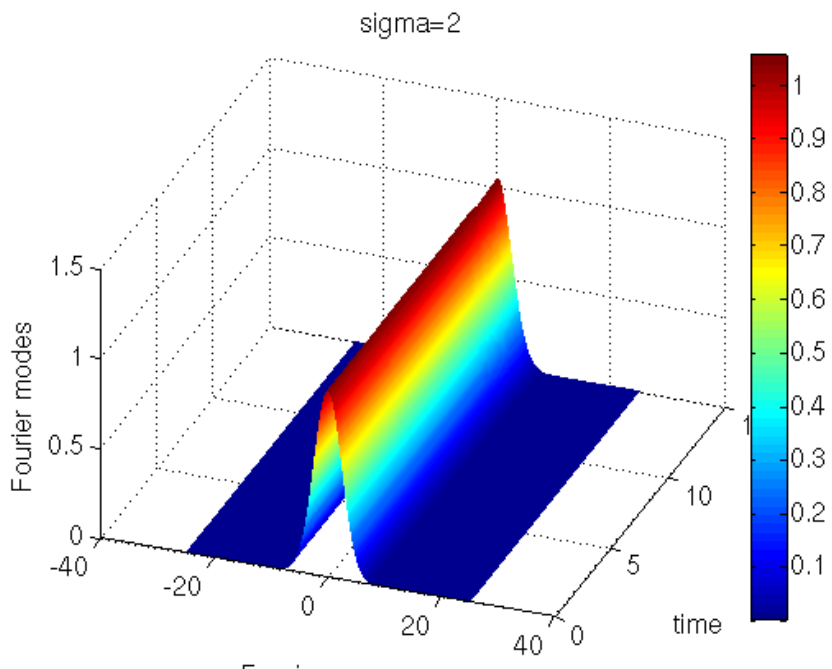

Fourier space

FIgURE 6. Space-time evolution of the modulus of the spatial spectrum of the solution; left : $\sigma=1$, right : $\sigma=2$.

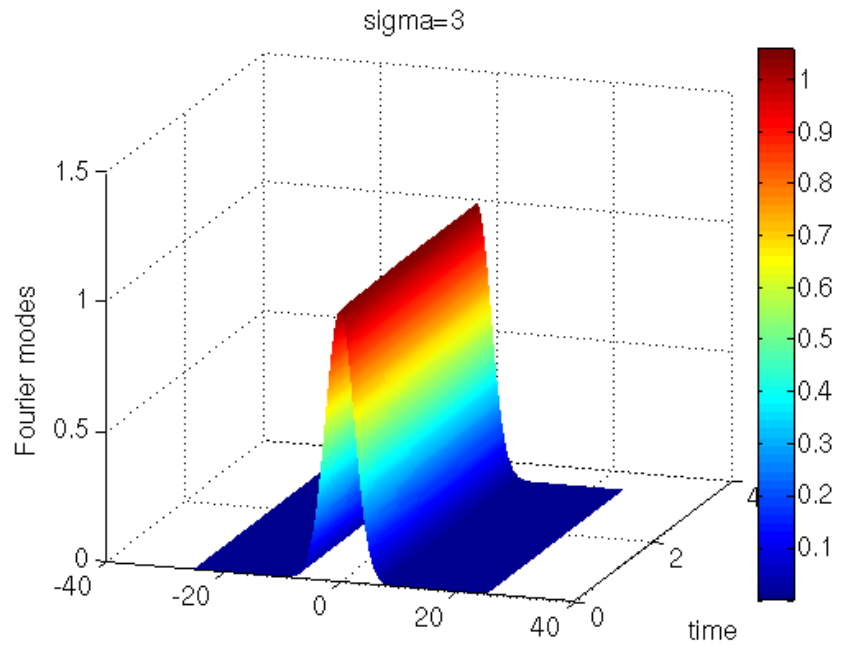

Fourier space

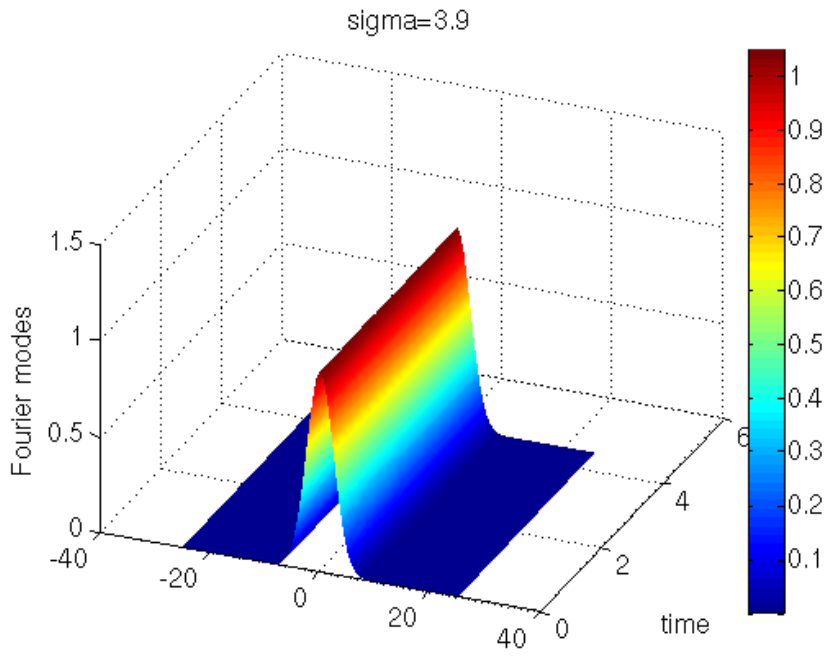

Fourier space

Figure 7. Space-time evolution of the modulus of the spatial spectrum of the solution; left : $\sigma=3$, right : $\sigma=3.9$.

approximates the mathematical expectation :

$$
<\max _{x}|\phi(t, x)|>=\frac{1}{N} \sum_{p=1}^{N} \max _{i}\left|\phi^{p}\left(t, x_{i}\right)\right|
$$



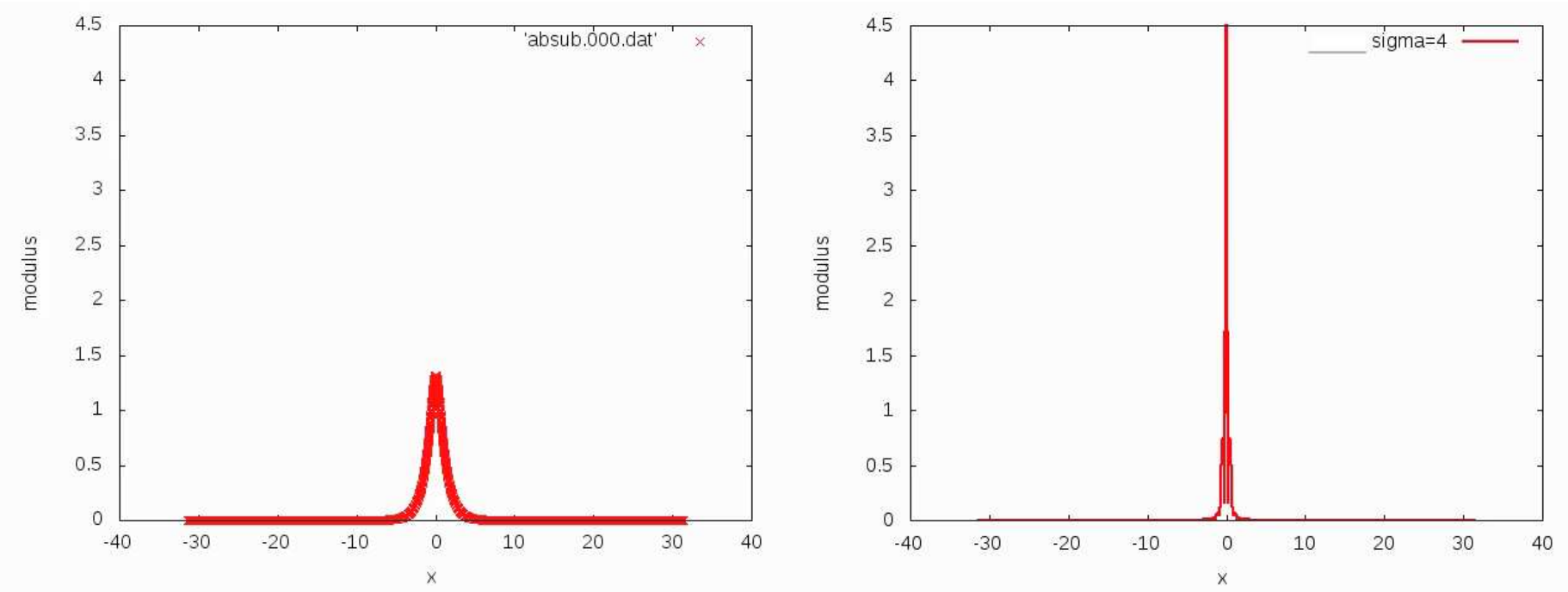

Figure 8. $\sigma=4$. Modulus of the initial data (left) and modulus of solution at time $t=0.035$ (right).

is the numerical approximation of the the average amplitude $\mathbb{E}\left(\max _{x}|\phi(t, x)|\right)$ where $N$ is number of computed trajectories. For the numerical simulation we take a Gaussian inital data given by $\phi(0, x)=\exp \left(-3 x^{2}\right)$ on the computational domain $\left[-L_{x}, L_{x}\right]=$ $[-20 \pi, 20 \pi]$ with $N_{x}=2^{12}$ points in space and a time step $\delta t=0.4 \delta x^{2}$ with a final time $T=1$. The average is taken over 1000 trajectories. There seems to be a rather strong diffusion of the expected amplitude as time evolves, but no clear time scale for the diffusion phenomenon can be extracted from the numerical simulations.

Acknowledgement : This work was supported by the ANR project STOSYMAP (ANR-2011-BS01-015-03). 

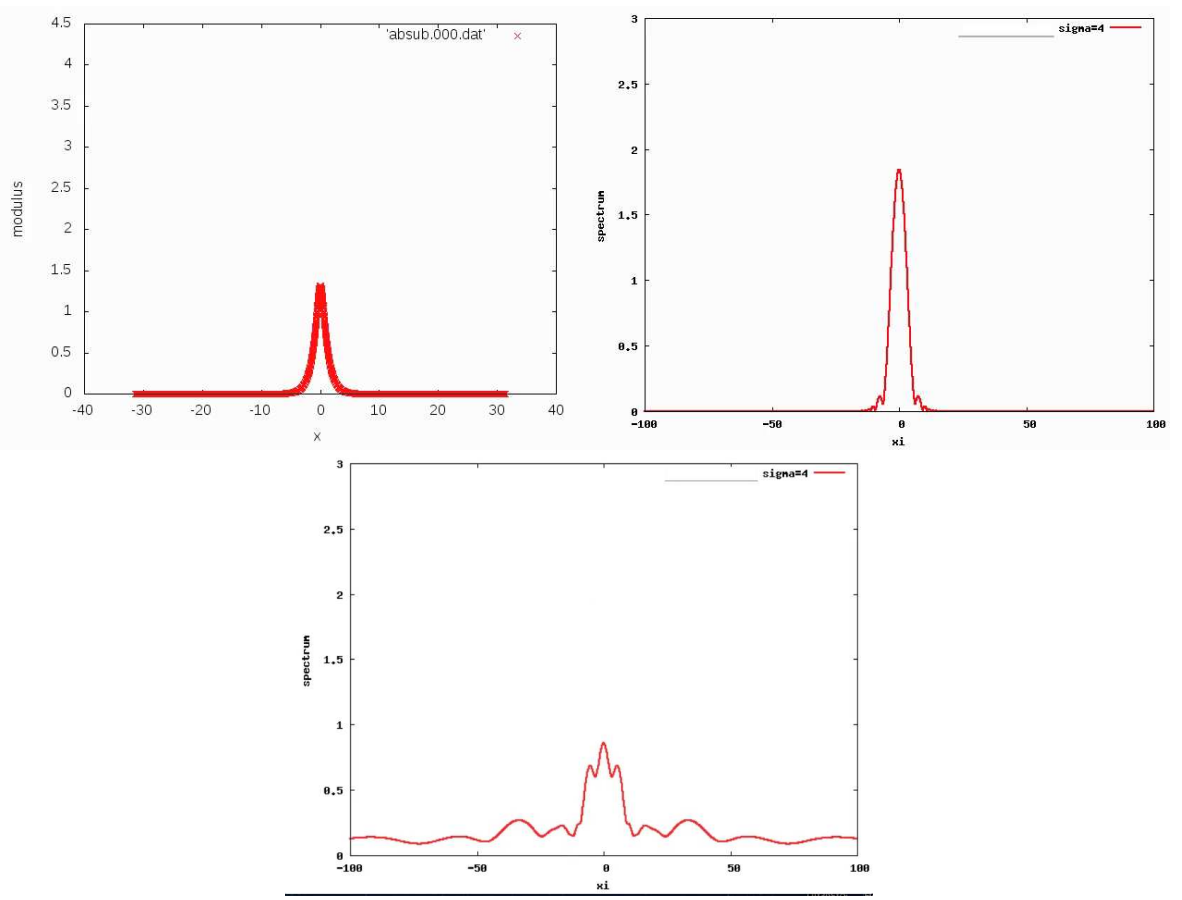

Figure 9. $\sigma=4$. Evolution of the spatial spectrum of the solution at times $t=0, t=0.00012$ and $t=0.035$.

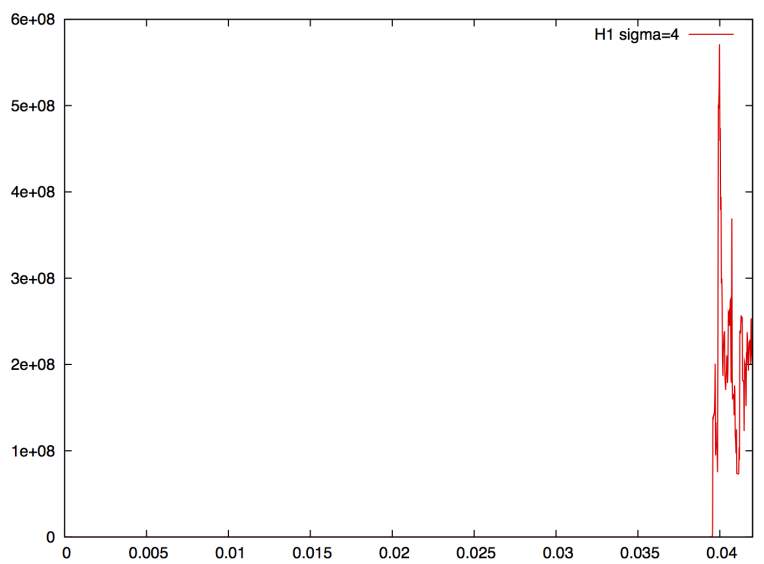

Figure 10. $\sigma=4$ : Evolution of the discrete $H^{1}$ norm.

\section{REFERENCES}

[1] G.P. Agrawal, Nonlinear fiber optics, 3rd ed.. Academic Press, San Diego, 2001. 


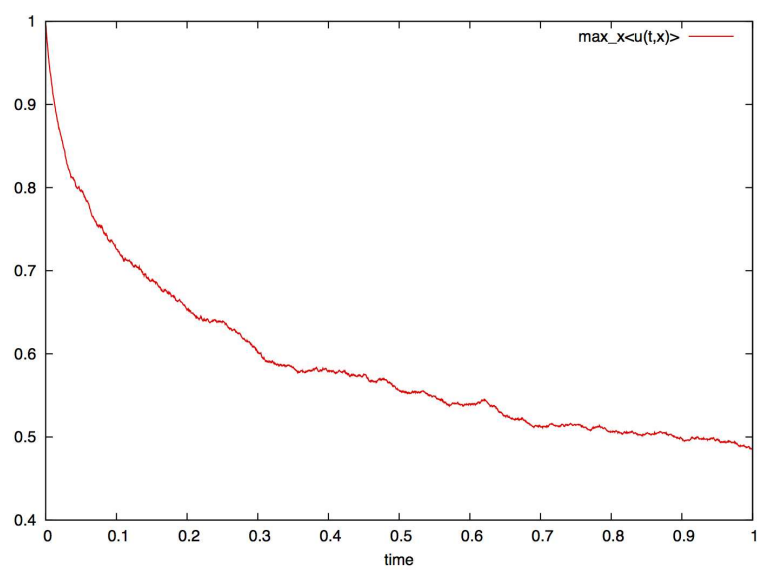

Figure 11. $\sigma=1$ : Time evolution of $<\max _{x}|\phi(t, x)|>$.

[2] G.P. Agrawal, Applications of nonlinear fiber optics, Academic Press, San Diego, 2001.

[3] M. Barton-Smith, A. Debussche, L. DiMenza Numerical study of two dimensional stochastic NLS equations, Num. Meth. for PDEs, 21, p.810-842, 2005.

[4] C. Besse. Schéma de relaxation pour l'équation de Schrödinger non linéaire et les systèmes de Davey et Stewartson. C.R. Acad. Sci. Paris. Sér. I Math., Vol. 326, (1998), 1427-1432.

[5] C. Besse , B. Bidgaray et S. Descombes. Order estimates in time of splitting methods for the nonlinear Schrödinger equation, SIAM J. Numer. Anal., Vol. 40, No. 1, (2002), pp 26-40.

[6] A. de Bouard, A. Debussche, A semi-discrete scheme for the stochastic nonlinear Schrödinger equation, Numer. Math. 96, n4, p.733-770, 2004.

[7] A. de Bouard, A. Debussche Blow-up for the supercritical stochastic nonlinear Schrödinger equation with multiplicative noise, Ann. Probab., 33, no. 3, p. 1078-1110, 2005.

[8] A. de Bouard, A. Debussche Weak and Strong Order of Convergence of a Semidiscrete Scheme for the Stochastic Nonlinear Schrödinger Equation, Appl. Math. Opt. 54, p. 369-399, 2006.

[9] A. de Bouard, A. Debussche The nonlinear Schrödinger equation with white noise dispersion, J. Funct. Anal. 259, no. 5, p. 1300-1321, 2010.

[10] A. Debussche, L. DiMenza Numerical simulation of focusing stochastic nonlinear Schrödinger equations, Physica D, 162 (3-4), p. 131-154, 2002.

[11] A. Debussche, Y. Tsutsumi 1-D quintic nonlinear Schrödinger equation with white noise dispersion, J. Math. Pures et Appl., 96, p. 363-376, 2011.

[12] M. Delfour, M. Fortin, G. Payre Finite difference solutions of a nonlinear Schrödinger equation, J. Comput. Phys. 44, no. 2, p. 277-288, 1981.

[13] R. Duboscq Analyse et simulations numriques d'quations de Schrdinger dterministes et stochastiques. Applications aux condensats de Bose-Einstein en rotation, thse de doctorat, Universit de Lorraine, 2013.

[14] O. Faure Simulation du mouvement brownien et des diffusions, thèse de doctorat, Ecole Nationale des Ponts et Chaussées, 1992.

[15] J. Garnier Stabilization of dispersion managed solitons in random optical fibers by strong dispersion management, Opt. Commun. 206, p. 411438, 2002. 
[16] M. Gazeau, Strong order of convergence of a semidiscrete scheme for the stochastic Manakov equation, preprint arxiv:1308.1576

[17] M. Kunze The singular perturbation limit of a variational problem from nonlinear fiber optics, Phys. D 180 (2003), no. 1-2, 108-114.

[18] R. Marty On a splitting scheme for the nonlinear Schrödinger equation in a random medium, Commun. Math. Sci. 4, no. 4, p. 679-705, 2006.

[19] G.N. Milstein, Approximate integration of stochastic differential equations, Theory Probab. Appl. 19, p. 583-588, 1974.

[20] J.M. Sanz-Serna, Methods for the numerical solution of the nonlinear Schrödinger equation, Math. Comput. 43, p. 21-27, 1984.

[21] S. Turitsyn, Path-averaged chirped optical soliton in dispersion-managed fiber communication lines, Opt. Comm. 163, p. 122-158, 1999.

[22] V. Zharnitsky, E. Grenier, C. Jones, S. Turitsyn, Stabilizing effects of dispersion management, Phys. D 152/153, p. 794-817, 2001.

Centre de Mathématiques Appliquées, CNRS et Ecole Polytechnique, 91128 Palaiseau CEDEX, FrANCE

E-mail address: belaouar@cmap.polytechnique.fr

E-mail address: debouard@cmap.polytechnique.fr

IRmar et Ens de Cachan, Antenne de Bretagne, Campus de Ker Lann, Av. R. SchuMAN, 35170 BRUZ, FRANCE

E-mail address: arnaud.debussche@bretagne.ens-cachan.fr 